\title{
Robust topology optimization accounting for spatially varying manufacturing errors
}

\author{
M. Schevenels ${ }^{\mathrm{a}}$, B.S. Lazarov ${ }^{\mathrm{b}}$, O. Sigmund ${ }^{\mathrm{b}}$ \\ ${ }^{a}$ Department of Civil Engineering, K.U.Leuven, Kasteelpark Arenberg 40, B-3001 Leuven, \\ Belgium \\ ${ }^{b}$ Department of Mechanical Engineering, Solid Mechanics, Technical University of \\ Denmark, Nils Koppels Alle, B. 404, 2800, Lyngby, Denmark
}

\begin{abstract}
This paper presents a robust approach for the design of macro-, micro-, or nanostructures by means of topology optimization, accounting for spatially varying manufacturing errors. The focus is on structures produced by milling or etching; in this case over- or under-etching may cause parts of the structure to become thinner or thicker than intended. This type of errors is modeled by means of a projection technique: a density filter is applied, followed by a Heaviside projection, using a low projection threshold to simulate under-etching and a high projection threshold to simulate over-etching. In order to simulate the spatial variation of the manufacturing error, the projection threshold is represented by a (non-Gaussian) random field. The random field is obtained as a memoryless transformation of an underlying Gaussian field, which is discretized by means of an EOLE expansion. The robust optimization problem is formulated in a probabilistic way: the objective function is defined as a weighted sum of the mean value and the standard deviation of the structural performance. The optimization problem is solved by means of a Monte Carlo method: in each iteration of the optimization scheme, a Monte Carlo simulation is performed, considering 100 random realizations of the manufacturing error. A more thorough Monte Carlo simulation with 10000 realizations is performed to verify the results obtained for the final design. The proposed methodology is successfully applied to two test problems: the design of a compliant mechanism and a heat conduction problem.
\end{abstract}

Keywords: Topology optimization, robust design optimization, manufacturing errors, Monte Carlo method

\section{Introduction}

The use of numerical optimization as a design tool has become widely spread in many areas of engineering. It supports the designer in finding the best com-

Email address: mattias.schevenels@bwk.kuleuven.be (M. Schevenels)

Preprint submitted to CMAME

1 July 2011

Published version: http://dx.doi.org/10.1016/j.cma.2011.08.006 
promise between cost and performance. Usually, a distinction is made between size, shape, and topology optimization. Size optimization is the most basic and widely used approach: the layout of the structure is fixed by the designer and the member dimensions (bar thicknesses) are determined by means of optimization. In shape optimization, the geometry is described by means of a number of parameters and the optimal parameter values are determined. The number of parameters can be very low (e.g. a single parameter describing the height of a truss) or very high (e.g. all node coordinates of a finite element model representing a shell structure). In the first case, the designer has a large degree of control, and the design space (i.e. the search space for the optimizer) is limited; in the second case, the opposite holds. Topology optimization does not require parameterization: the designer specifies the design domain (in physical space), the loads and support conditions, and the amount of available material, and the optimal distribution of material is determined [4, 5]. Compared to shape optimization, this approach implies a much larger design space, as the type of structure is not fixed a priori. For this reason, topology optimization has a large potential as a design tool in the early design phase.

The performance of a structure is almost always subject to a number of uncertain parameters, such as variations of the material properties, unknown loading and support conditions, and geometric imperfections. The effect of these uncertainties may be significant, resulting in a large deviation between the performance of the optimized design and the actual structure. In a recent paper, Schüeller and Jensen give an overview of methods to account for uncertainties in optimization [33].

One of the most popular strategies to account for uncertainties is robust design optimization. The aim of robust design optimization is to maximize the design's performance while simultaneously minimizing its sensitivity with respect to uncertainties. A robust design optimization problem can be formulated in various ways - see reference [6] for a comprehensive overview. A worst case approach is often followed, where the uncertain parameters are allowed to vary in a specified interval and the structure with the worst performance is considered in the optimization. Alternatively, a probabilistic approach can be followed, where the uncertain parameters are assigned a probability distribution and the objective function is defined as a weighted average of the mean value and the standard deviation of the structural performance.

While the use of a robust approach for size and shape optimization has been extensively studied, publications on robust topology optimization are still relatively scarce. The earliest publications in this field address the design of truss structures. Ben-Tal and Nemirovski [3] propose a method based on semi-definite programming for robust truss topology optimization accounting for uncertain load conditions. Sandgren and Cameron [31] address robust truss topology optimization considering uncertain variations of the load, the geometry, and the material properties. Seepersad et al. [34] propose a robust design method for cellular materials on a mesoscopic scale. The problem is formulated by means of a ground structure, in a similar way as in truss topology design problems. The aim is to achieve robustness with respect to topological imperfections (errors 
in cellular connectivity) and dimensional imperfections (variations of cell wall thickness).

In later publications, optimal material distribution problems are considered. Kogiso et al. [23] focus on the design of compliant mechanisms, accounting for uncertain variations of the direction of the driving force. De Gournay et al. [10] use the level set method for the design of robust structures with minimum compliance considering uncertain loads. Chen et al. [9] also use the level set method for the design of robust structures with minimum compliance and for the design of robust compliant mechanisms. They consider uncertainties in the loading pattern and the material properties. The uncertain parameters are assumed to vary in space and modeled as random fields.

Other authors follow a reliability based approach to topology optimization $[21,29,39]$. In reliability based design optimization, the focus is on the failure probability of the structure rather than its sensitivity with respect to uncertainties. The optimization problem is usually formulated as a maximum (expected) performance problem with a probabilistic constraint in order to ensure that the failure probability of the structure remains below an acceptable level.

Sigmund [37] and Wang et al. [43] present a robust topology optimization scheme accounting for uniform manufacturing errors. This method is developed for the design of structures produced by milling or etching, such as MicroElectro-Mechanical Systems (MEMS). The focus is on errors caused by uniform over-etching (all parts of the structure are thinner than intended) or underetching (all parts are thicker than intended). The effect of over- and underetching is simulated by means of a projection method $[17,36,45]$ : the design is smoothed by means of a density filter, and a Heaviside projection is applied, using a high projection threshold to simulate over-etching and a low projection threshold to simulate under-etching. The application of this method to the design of photonic crystal waveguides is elaborated in reference [42]. A similar approach is followed for the design of large-displacement compliant mechanisms in reference [25].

Depending on the production process, it may be more realistic to assume that the manufacturing error is non-uniform, i.e. that the magnitude of the over- or under-etching error varies randomly throughout the design domain. The present paper therefore extends the method proposed by Sigmund [37] and Wang et al. [43] to the case of non-uniform manufacturing errors. To this end, the Heaviside projection threshold is modeled as a random field. The method is applied to two example problems: the design of a compliant gripper mechanism (figure 1) and the design of a heat sink (figure 3).

The paper is organized as follows. Section 2 focuses on the deterministic topology optimization problem. The SIMP method and the use of a Heaviside projection technique are briefly reviewed, and the example problems are introduced. Section 3 addresses the method proposed by Sigmund [37] to model the effect of uniform over- and under-etching and extends this method to the non-uniform case, i.e. the case where the manufacturing error varies randomly in space. In section 4, the robust topology optimization problem is formulated and solved for the two example problems. The designs obtained by robust op- 
timization (assuming either uniform or non-uniform manufacturing errors) are compared with the designs obtained by deterministic optimization. Finally, in section 5, the conclusions of the paper are summarized.

\section{Deterministic topology optimization}

\subsection{SIMP with Heaviside projection filtering}

A widely used method for topology optimization is the SIMP method, which is based on the use of a Solid Isotropic Material with Penalization [5]: the design domain is subdivided using finite elements, and each element $e$ is assigned a density $\overline{\tilde{\rho}}_{e}$ representing the presence of material (zero density for void, unit density for solid elements). Intermediate densities are also allowed in order to obtain a continuous optimization problem, but they are penalized in order to avoid intermediate densities in the final design. The penalization is realized by means of a nonlinear relation between an element's density $\overline{\tilde{\rho}}_{e}$ and its stiffness or conductivity $E_{e}$ :

$$
E_{e}\left(\overline{\tilde{\rho}}_{e}\right)=E_{\min }+\overline{\tilde{\rho}}_{e}^{p}\left(E_{0}-E_{\min }\right)
$$

$E_{0}$ and $E_{\min }$ are the stiffness/conductivity of the solid phase and the void phase, respectively, and $p$ is a penalization factor used to ensure black-and-white solutions.

Using the element densities $\overline{\tilde{\rho}}_{e}$ as design variables in the optimization problem would lead to a mesh dependent solution and to the formation of checkerboard patterns $[11,19,38]$. A common technique to avoid these problems is the use of a density filter $[7,8]$ : the density of an element is defined as a weighted average of the design variables corresponding to the elements in its neighborhood. The neighborhood is defined as a circle in $2 \mathrm{D}$ or a sphere in $3 \mathrm{D}$ with a specified radius $R$. The filter radius $R$ determines the minimum length scale in the optimized design. The application of a density filter can be interpreted as a smoothing operation and leads to gray transition zones between solid and void regions. Projection techniques have been proposed to transform the filtered design into a black-and-white solution [17, 20, 36, 45].

The design variables are denoted as $\rho_{e}$. The application of a density filter leads to the variables $\tilde{\rho}_{e}$ :

$$
\tilde{\rho}_{e}=\frac{\sum_{i=1}^{Q} w_{e i} v_{i} \rho_{i}}{\sum_{i=1}^{Q} w_{e i} v_{i}}
$$

where $Q$ is the number of elements, $r_{e i}$ is the center-to-center distance between elements $e$ and $i$, and and $w_{e i}$ is a weighting factor defined as:

$$
w_{e i}=\max \left(0, R-r_{e i}\right)
$$

The filtered variables $\tilde{\rho}_{e}$ are further transformed into element densities $\overline{\tilde{\rho}}_{e}$ by means of a Heaviside step function: values smaller than a threshold value $\eta$ are projected to 0 ; values larger than $\eta$ are projected to 1 . In order to allow for 
the use of a gradient-based optimization scheme, the Heaviside step function is replaced with a smooth approximation. The function proposed by Wang et al. [43] is used:

$$
\overline{\tilde{\rho}}_{e}=\frac{\tanh (\beta \eta)+\tanh \left(\beta\left(\tilde{\rho}_{e}-\eta\right)\right)}{\tanh (\beta \eta)+\tanh (\beta(1-\eta))}
$$

The parameter $\beta$ controls the smoothness of the approximation: for $\beta$ tending to zero, the element densities $\overline{\tilde{\rho}}_{e}$ are equal to the filtered variables $\tilde{\rho}_{e}$; for $\beta$ approaching infinity, the approximation approaches a true Heaviside step function. In order to avoid local minima in the optimization, a continuation scheme is used: the parameter $\beta$ starts from a low value and is gradually increased during the optimization process.

In the following sections, the design variables $\rho_{e}$, the filtered variables $\tilde{\rho}_{e}$, and the element densities are collected in vectors denoted as $\boldsymbol{\rho}, \tilde{\boldsymbol{\rho}}$, and $\overline{\tilde{\boldsymbol{\rho}}}$, respectively. The dependencies between these vectors and the relation with the Heaviside projection threshold $\eta$ are made explicit by means of the notations $\tilde{\boldsymbol{\rho}}(\boldsymbol{\rho})$ and $\overline{\tilde{\rho}}(\tilde{\boldsymbol{\rho}} \mid \eta)$.

\subsection{Formulation of the optimization problem}

The optimization problem is formulated as follows:

$$
\begin{aligned}
\min _{\boldsymbol{\rho}} & : f(\boldsymbol{\rho})=\mathbf{1}^{\mathrm{T}} \mathbf{u}(\boldsymbol{\rho}) \\
\text { s.t. } & : \mathbf{K}(\boldsymbol{\rho}) \mathbf{u}(\boldsymbol{\rho})=\mathbf{f} \\
& : g(\boldsymbol{\rho})=V(\boldsymbol{\rho}) \leq V^{*} \\
& : \mathbf{0} \leq \boldsymbol{\rho} \leq \mathbf{1}
\end{aligned}
$$

where $\mathbf{K u}=\mathbf{f}$ is the discrete version of the state problem, i.e. the discrete Navier-Cauchy partial differential equation for the mechanism design problem and the discrete Poisson equation for the heat conduction problem. The vectors $\mathbf{f}$ and $\mathbf{u}$ are discrete representations of the load and the solution, respectively. The matrix $\mathbf{K}$ is obtained by finite element discretization of the differential operator. It is assembled from the element stiffness matrices $\mathbf{K}_{e}=E_{e} \mathbf{K}_{0}$, where $\mathbf{K}_{0}$ is the element stiffness matrix for an element with unit stiffness/conductivity. The objective function $f(\boldsymbol{\rho})$ is expressed in terms of the elements of the vector $\mathbf{u}$ by means of a problem dependent selection vector $\mathbf{l}$. For the compliant mechanism problem, the elements of the selection vector 1 corresponding to the upper and lower output degrees of freedom are -1 and 1, respectively, and all other elements are equal to zero. For the heat conduction problem, the selection vector $\mathbf{l}$ is equal to the load vector $\mathbf{f}$, which is an evenly distributed heat generation load. The design variables $\rho_{e}$ are collected in a vector $\boldsymbol{\rho}$ and must take a value between 0 and 1 . The volume fraction $V(\boldsymbol{\rho})$ of the design domain occupied by material is given by:

$$
V(\boldsymbol{\rho})=\frac{\sum_{e=1}^{Q} \overline{\tilde{\rho}}_{e} v_{e}}{\sum_{e=1}^{Q} v_{e}}
$$


where $v_{e}$ is the volume of element $e$. The maximum volume fraction is $V^{*}$.

The sensitivity $\frac{\partial f}{\partial \tilde{\rho}_{e}}$ of the objective function $f$ with respect to the element densities $\overline{\tilde{\rho}}_{e}$ is computed by means of the adjoint variable method:

$$
\frac{\partial f}{\partial \tilde{\tilde{\rho}}_{e}}=-\boldsymbol{\lambda}^{\mathrm{T}} \frac{\partial \mathbf{K}}{\partial \tilde{\tilde{\rho}}_{e}} \mathbf{u}
$$

where the adjoint vector $\boldsymbol{\lambda}$ is obtained by solving $\mathbf{K} \boldsymbol{\lambda}=1$. For the heat conduction problem, the adjoint vector $\boldsymbol{\lambda}$ is equal to the state vector $\mathbf{u}$.

The sensitivity $\frac{\partial g}{\partial \tilde{\rho}_{e}}$ of the constraint function $g$ with respect to the element densities $\overline{\tilde{\rho}}_{e}$ is given by:

$$
\frac{\partial g}{\partial \tilde{\tilde{\rho}}_{e}}=\frac{v_{e}}{\sum_{i=1}^{Q} v_{i}}
$$

The sensitivity $\frac{\partial f}{\partial \rho_{k}}$ of the objective function $f$ with respect to the design variables $\rho_{k}$ is obtained by applying the chain rule twice:

$$
\frac{\partial f}{\partial \rho_{k}}=\sum_{e=1}^{Q} \frac{\partial f}{\partial \tilde{\rho}_{e}} \frac{\partial \tilde{\rho}_{e}}{\partial \rho_{k}}=\sum_{e=1}^{Q} \frac{\partial f}{\partial \tilde{\tilde{\rho}}_{e}} \frac{\partial \overline{\tilde{\rho}}_{e}}{\partial \tilde{\rho}_{e}} \frac{\partial \tilde{\rho}_{e}}{\partial \rho_{k}}
$$

where:

$$
\frac{\partial \tilde{\rho}_{e}}{\partial \rho_{k}}=\frac{w_{e k} v_{k}}{\sum_{i=1}^{Q} w_{e i} v_{i}}
$$

and:

$$
\frac{\partial \overline{\tilde{\rho}}_{e}}{\partial \tilde{\rho}_{e}}=\frac{\beta\left(\operatorname{sech}\left(\beta\left(\tilde{\rho}_{e}-\eta\right)\right)\right)^{2}}{\tanh (\beta \eta)+\tanh (\beta(1-\eta))}
$$

The sensitivity $\frac{\partial g}{\partial \rho_{k}}$ of the constraint function $g$ with respect to the design variables $\rho_{k}$ is obtained in a similar way.

\subsection{Compliant mechanism design}

The design domain and the boundary conditions for the compliant gripper mechanism are given in figure 1 . The objective is to find a symmetric design (with respect to the horizontal axis) that maximizes the sum of the displacements $u_{\text {out } 1}$ and $u_{\text {out } 2}$ at the output points for a unit force $f_{\text {in }}=1$ applied at the input point. The width and height of the design domain is $L=200$. The spring stiffness coefficients are $k_{\mathrm{in}}=1$ and $k_{\text {out }}=0.005$. The Young's moduli for the solid and the void phases are $E_{0}=1$ and $E_{\min }=10^{-9}$, respectively. The Poisson's ratio is $\nu=0.3$. The maximum volume fraction is $V^{*}=0.3$. A finite element mesh consisting of $200 \times 200$ linear elements is used.

The SIMP penalization factor is $p=3$. The filter radius of the density filter is $R=8.4$. Following $\mathrm{Xu}$ et al. [45] and Kawamoto et al. [20], a Heaviside projection threshold $\eta$ equal to 0.5 is used. The initial value for the smoothness parameter $\beta$ is 1 ; this value is doubled every 50 iterations up to a maximum value of 32. The Method of Moving Asymptotes (MMA) developed by Svanberg [41] is used to solve the optimization problem. The optimization algorithm is 


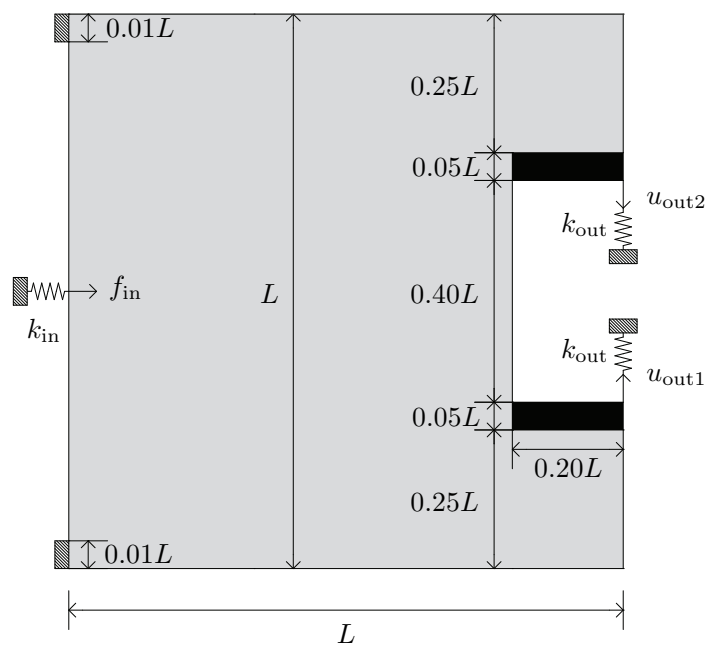

Figure 1: Design domain and boundary conditions for the compliant gripper mechanism.

terminated after 300 iterations. This termination criterion (with a fixed number of iterations) is used to keep the computational time limited and predictable, which is especially useful in the (computationally demanding) robust approach addressed in the following sections. For all optimization runs discussed in this paper, the relative change of the objective function in the final iteration is smaller than 0.0002 . While the $l^{\infty}$ norm of the change of the design variables remains relatively large (up to 0.17 for one of the optimization runs), we have the experience that increasing the number of iterations only has a minor influence on the design and practically no influence on the objective function.

Figure 2 shows the optimized design. The design variables $\boldsymbol{\rho}$, the intermediate variables $\tilde{\boldsymbol{\rho}}$ obtained by density filtering, and the element densities $\overline{\tilde{\boldsymbol{\rho}}}$ obtained by a (smoothed) Heaviside projection are shown. It is important to note that only the element densities $\overline{\tilde{\rho}}$ have a physical meaning, as they determine the stiffness taken into account in the finite element analysis. The value of the objective function for the optimized design is $f(\boldsymbol{\rho})=-2.023$.

While the application of the density filter introduces a length scale in the design, this length scale is not preserved by the Heaviside projection: very narrow hinges occur between different parts of the mechanism. As explained by Wang et al. [43], this is caused by the use of a projection threshold $\beta$ different from 0 or 1 . Due to these hinges, the design is very sensitive to manufacturing errors, as they may cause the parts of the mechanism to disconnect in the case of over-etching.

\subsection{Heat sink design}

Figure 3 shows the design domain and boundary conditions for the heat sink. The heat generation load is an evenly distributed unit load, and the objective is to maximize the heat transfer. The width and height of the design domain is 


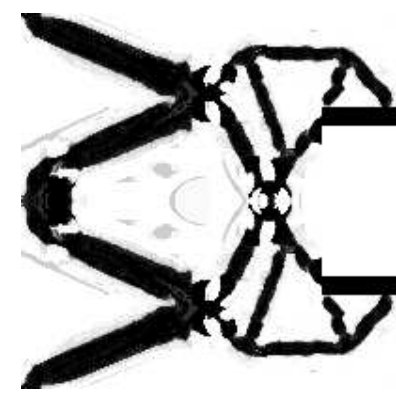

(a) Design variables $\rho$.

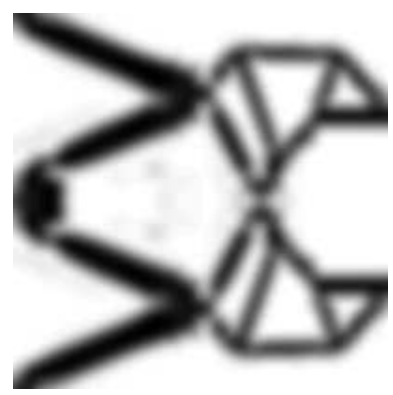

(b) Filtered variables $\tilde{\boldsymbol{\rho}}(\boldsymbol{\rho})$.

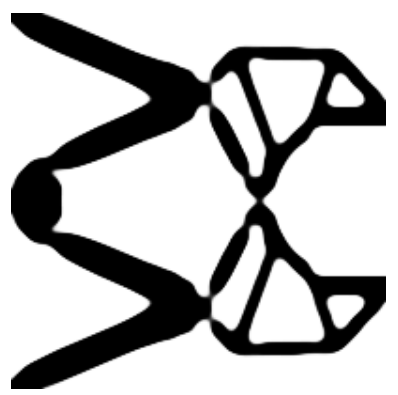

(c) Element densities $\overline{\tilde{\boldsymbol{\rho}}}(\tilde{\boldsymbol{\rho}} \mid \eta)$.

Figure 2: Optimized design for a compliant gripper mechanism obtained with a deterministic approach.

$L=200$. The conductivity of the solid phase is $E_{0}=1$, while the conductivity of the void phase is $E_{\min }=10^{-3}$. The maximum volume fraction is $V^{*}=0.5$. A finite element mesh with $200 \times 200$ elements is used.

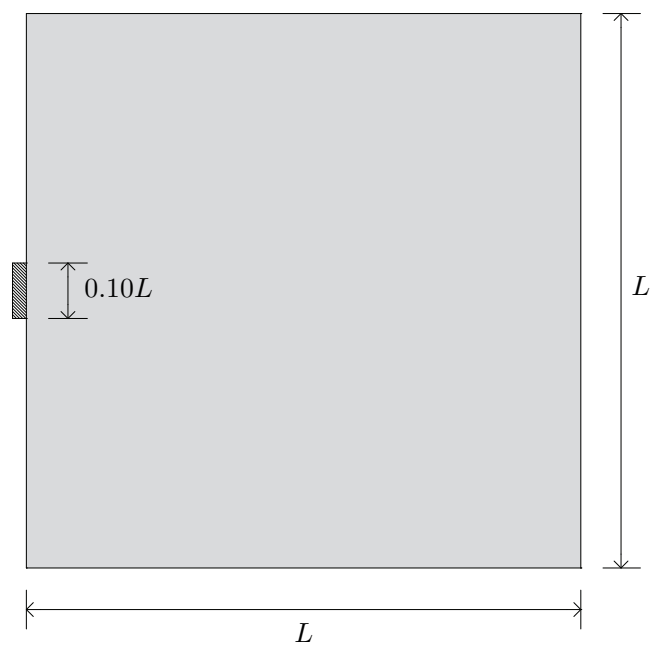

Figure 3: Design domain and boundary conditions for the heat sink. A zero temperature is imposed at the hashed area, while a zero heat flux is imposed elsewhere.

As in the previous example, the SIMP penalization factor is $p=3$, the filter radius is $R=8.4$, the Heaviside projection threshold is $\eta=0.5$, and the smoothness parameter $\beta$ is increased from 1 to 32 by doubling its value every 50 iterations. MMA is used, and the optimization algorithm is stopped after 300 iterations.

The optimized design is shown in figure 4 . The value of the objective function for this design is $f(\boldsymbol{\rho})=1.179$. As stated by Wang et al. [43], the use of a Heaviside projection filter with a threshold $\eta=0.5$ does not lead to a design 
with a minimum length scale, neither for the solid phase, nor for the void phase.

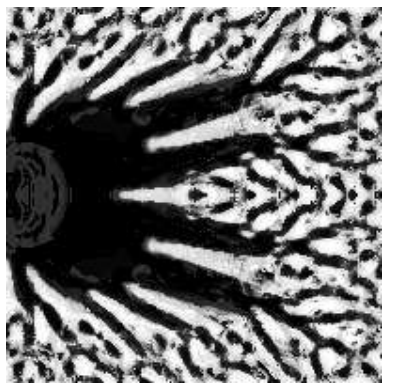

(a) Design variables $\rho$.

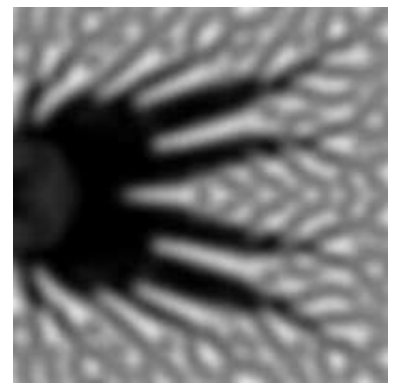

(b) Filtered variables $\tilde{\boldsymbol{\rho}}(\boldsymbol{\rho})$.

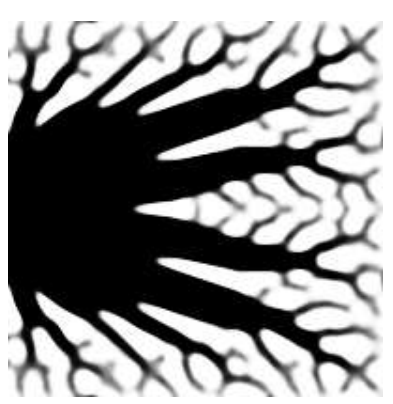

(c) Element densities $\overline{\tilde{\boldsymbol{\rho}}}(\tilde{\boldsymbol{\rho}} \mid \eta)$.

Figure 4: Optimized design for a heat sink obtained with a deterministic approach.

It should be noted that the design in figure $4 \mathrm{c}$ exhibits a relatively large amount of gray elements; as a consequence, this design is not immediately usable as input to the manufacturing process. It is possible to suppress these elements by increasing the smoothness parameter $\beta$ to a higher value, e.g. 128 or 256 . However, this leads to a structure with very fine features (a single element wide, see e.g. reference [43]), which is impossible to produce.

\section{Modeling manufacturing errors}

\subsection{Uniform errors}

Sigmund [37] and Wang et al. [43] propose a method to model the effect of over-etching (erosion) and under-etching (dilation) by varying the Heaviside projection threshold $\eta$. The basis of this idea is illustrated in figure 5 . The figure shows a design consisting of vertical bars. The design variables $\rho$ are smoothed by means of a density filter. The filtered design $\tilde{\boldsymbol{\rho}}$ exhibits gray transition zones between the solid and void regions. The application of a Heaviside projection leads to a black-and-white design. The reference design (i.e. the design to be used as input to the manufacturing process) is obtained with a projection threshold $\eta_{0}=0.5$. Varying the projection threshold $\eta$ results in an erosion or dilation effect: for high values of $\eta$, only dark gray elements are projected to the solid phase, resulting in thinner bars. For low values of $\eta$, light gray elements are also projected to the solid phase, and the bars become thicker. The effect is shown for three different projection thresholds represented as a shade of gray.

While Sigmund [37] and Wang et al. [43] follow a worst case approach to account for the effect of manufacturing errors in the optimization, a probabilistic approach is followed in this paper: the projection threshold is modeled as a random variable, characterized by a probability distribution. The motivation to switch from a worst case approach to a probabilistic approach is that the latter allows for a relatively straightforward extension of the method to non-uniform manufacturing errors by using a random field instead of a random variable. 

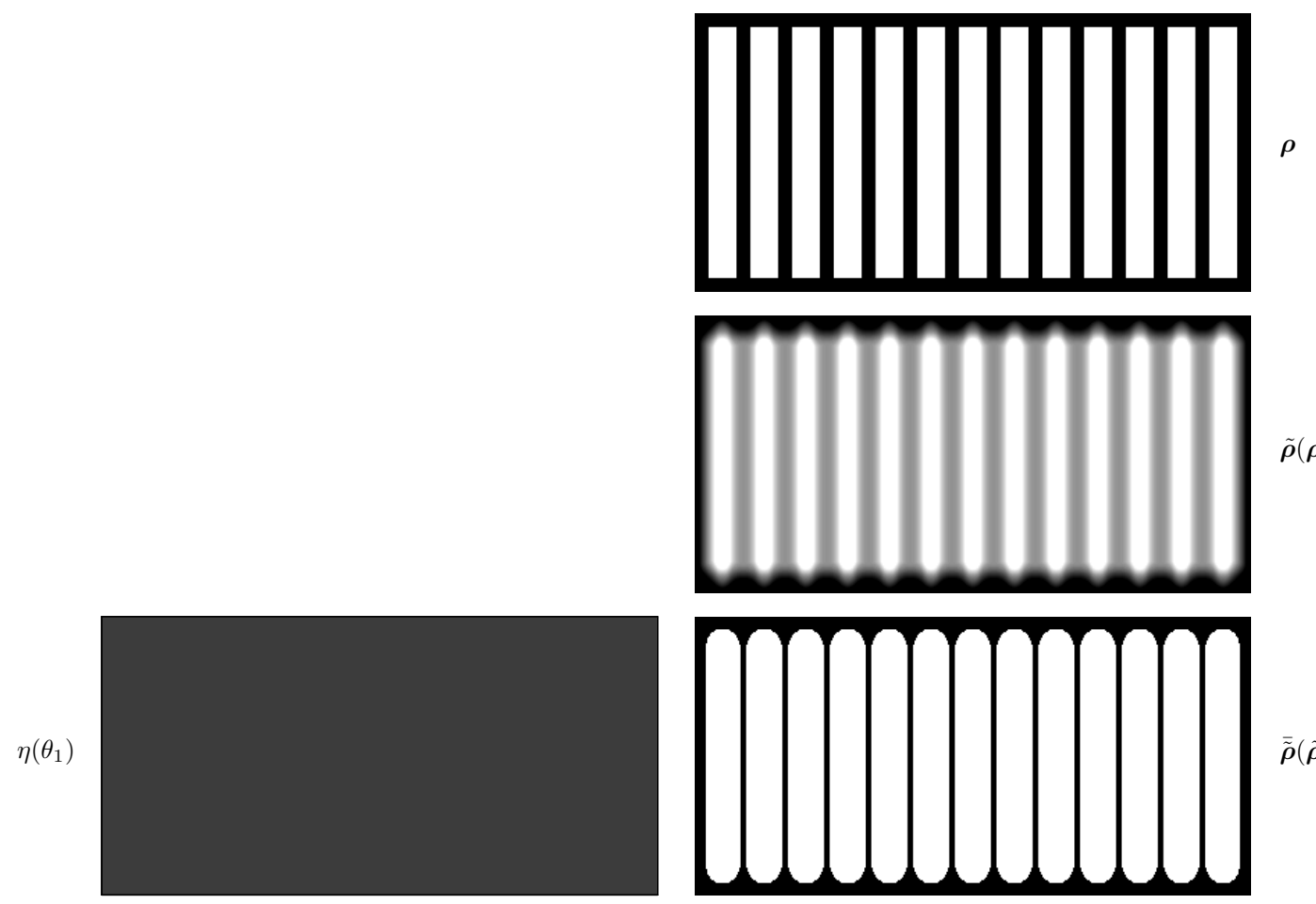

$\overline{\tilde{\boldsymbol{\rho}}}\left(\tilde{\boldsymbol{\rho}} \mid \eta\left(\theta_{1}\right)\right)$
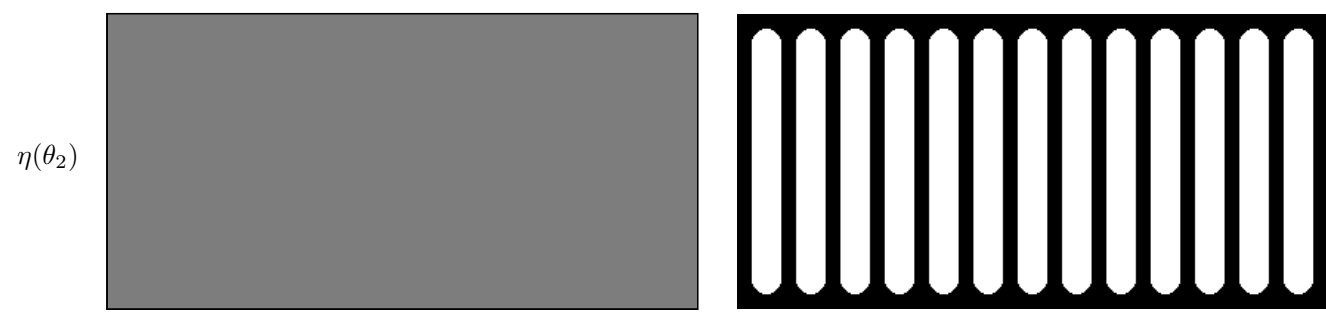

$\overline{\tilde{\boldsymbol{\rho}}}\left(\tilde{\boldsymbol{\rho}} \mid \eta\left(\theta_{2}\right)\right)$
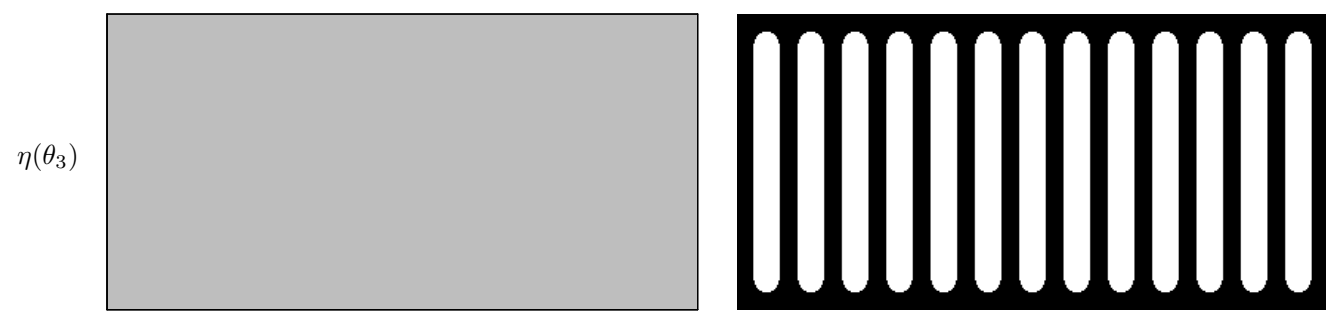

$\overline{\tilde{\boldsymbol{\rho}}}\left(\tilde{\boldsymbol{\rho}} \mid \eta\left(\theta_{3}\right)\right)$

Figure 5: Uniform manufacturing errors modeled by means of a random Heaviside projection threshold $\eta(\theta)$. The design variables $\boldsymbol{\rho}$, the intermediate variables $\tilde{\boldsymbol{\rho}}(\boldsymbol{\rho})$, and the element densities $\overline{\tilde{\boldsymbol{\rho}}}(\tilde{\boldsymbol{\rho}} \mid \eta(\theta))$ obtained for three random realizations $\eta\left(\theta_{1}\right), \eta\left(\theta_{2}\right)$, and $\eta\left(\theta_{3}\right)$ of the projection threshold are shown.

In Kolmogorov's probability theory [13, 24], a random variable $\eta$ is represented as a function $\eta(\theta)$, where $\theta$ stands for an elementary event. The ele- 
mentary event determines the state of the random problem: every elementary event corresponds to a single possible state. Within each state, all random variables considered assume a fixed, deterministic value. The random projection threshold $\eta(\theta)$ is characterized by a probability density function $p_{\eta}(\eta)$.

The Gaussian probability distribution is frequently adopted to characterize the uncertainty in mechanical models as this leads to tractable numerical formulations and allows for the use of relatively simple simulation models, especially when random fields are involved. This choice is often motivated by the central limit theorem: the uncertainties are assumed to originate from a large number of (independent) sources, and the sum of a large number of independent random variables tends to a Gaussian variable. However, the use of a Gaussian probability distribution is not always appropriate. In many publications, the stiffness of a mechanical system is modeled by means of a Gaussian random variable or field. This is clearly unrealistic, as in this case the stiffness takes a negative value with non-zero probability. Moreover, it can easily be shown that the variance of the response of such a system due to a static load becomes infinite, which is not physically sound [32]. Similarly, the Heaviside projection threshold $\eta(\theta)$ must take a value between 0 and 1 in order to be meaningful. It can therefore not be modeled as a Gaussian random variable. The probability density function $p_{\eta}(\eta)$ shown in figure 6 is used: the projection threshold $\eta(\theta)$ is assumed to be uniformly distributed in the interval from 0.4 to 0.6. As the reference design is the design corresponding to $\eta_{0}=0.5$, the choice of this probability density function implies that erosion and dilation errors are equally likely to occur, since the probability density function $p_{\eta}(\eta)$ is symmetric with respect to $\eta_{0}$.

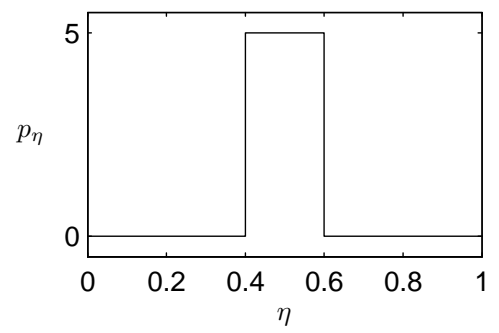

Figure 6: Probability density function $p_{\eta}(\eta)$ of the projection threshold $\eta(\theta)$.

In order to obtain a similar formulation for uniform and non-uniform manufacturing errors (treated in the next subsection), the projection threshold $\eta(\theta)$ is expressed as a nonlinear transformation of an underlying standard Gaussian variable $\xi(\theta)$ :

$$
\eta(\theta)=\gamma(\xi(\theta))=F_{\eta}^{-1}\left(F_{\xi}(\xi(\theta))\right)
$$

The transformation $\gamma$ is defined in terms of the cumulative distribution function $F_{\eta}(\eta)$ of the random variable $\eta(\theta)$ and the standard Gaussian cumulative distribution function $F_{\xi}(\xi)$. 
The combination of the density filter radius $R$ and the projection thresholds $\eta$ considered in the optimization determines the length scale of the solid and void phases as well as the magnitude of the manufacturing errors taken into account. The influence of $R$ and $\eta$ on the length scale $b$ is studied in reference [43]; this study is not repeated in the present paper. ${ }^{1}$ In the following, the relation between the projection threshold $\eta$ and the ratio $\varepsilon / R$ of the manufacturing error magnitude $\varepsilon$ and the filter radius $R$ is investigated.

Figure 7 illustrates the simulation of a manufacturing error for a square structure with a circular hole. The design variables $\rho$ are shown in figure 7 a. The application of a density filter leads to the variables $\tilde{\boldsymbol{\rho}}(\boldsymbol{\rho})$ shown in figure $7 \mathrm{~b}$. The radius $R$ of the filter determines the width of the gray transition zone between the solid and void phases, which is equal to $2 R$. The element densities $\overline{\tilde{\boldsymbol{\rho}}}(\tilde{\boldsymbol{\rho}} \mid \eta(\theta))$ are computed for three realizations $\eta\left(\theta_{1}\right), \eta\left(\theta_{2}\right)$, and $\eta\left(\theta_{3}\right)$ of the projection threshold, corresponding to an eroded, and intermediate, and a dilated design. The results are shown in figure $7 \mathrm{~b}$. As can be observed, the manufacturing errors are confined to the gray transition zone. If the projection threshold $\eta(\theta)$ ranges from 0 to 1 , the manufacturing errors cover the entire transition zone. If the range of the projection threshold $\eta(\theta)$ is narrower, the manufacturing error becomes smaller.

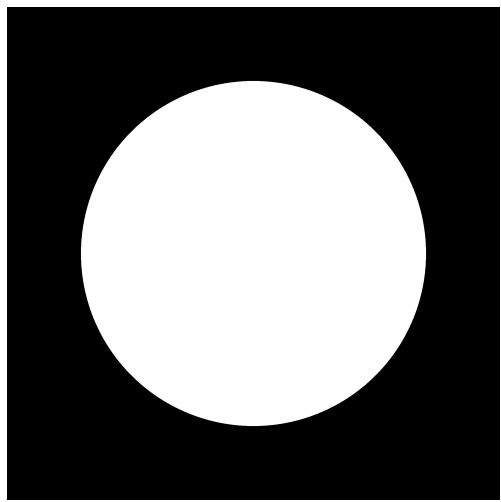

(a)

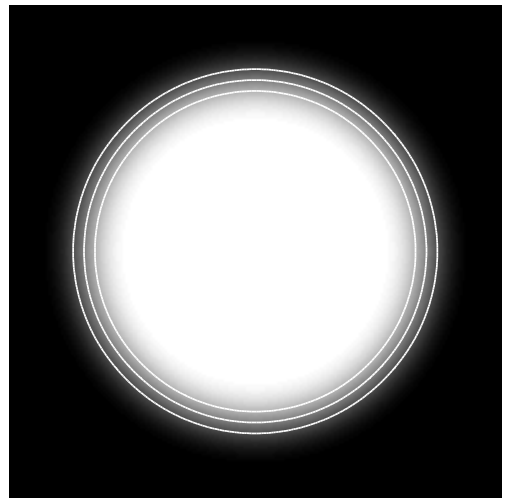

(b)

Figure 7: (a) Design variables $\rho$ for a square structure with a circular hole and (b) corresponding filtered variables $\tilde{\boldsymbol{\rho}}(\boldsymbol{\rho})$. The element densities $\overline{\tilde{\boldsymbol{\rho}}}(\tilde{\boldsymbol{\rho}} \mid \eta(\theta))$ are computed for three realizations $\eta\left(\theta_{1}\right), \eta\left(\theta_{2}\right)$, and $\eta\left(\theta_{3}\right)$ of the projection threshold. For each realization, the boundary between the solid and the void phase is indicated by means of a line.

Next, a quantitative study of the problem is performed. Figure 8 shows how a variation of the projection threshold $\eta$ leads to a shift of the boundary between a solid and a void phase for a 1D problem (or a straight/planar boundary for

\footnotetext{
${ }^{1}$ Figures 12 and 21 in reference [43] show the normalized length scale as a function of the projection threshold $\eta$. In these figures, the vertical axis is erroneously labeled as $b / 2 R$. The correct label is $b / R$, where $b$ is the length scale and $R$ is the filter radius.
} 
$2 \mathrm{D} / 3 \mathrm{D}$ problems). Two cases are considered: in the first case, the dimension of both the solid and the void phase is larger than $2 R$. In the second case, the dimension of the solid phase is smaller than $2 R$. The figure shows that increasing the projection threshold from $\eta_{0}=0.5$ to $\eta=0.6$ leads to an erosion effect. It can be observed that the magnitude $\varepsilon$ of the erosion is larger in the case where the dimension of the solid phase is smaller than $2 R$. This is due to the fact that the application of a density filter results in a curve with a smaller slope. Analogously, a decrease of the projection threshold leads to a dilation which is stronger in the case where the dimension of the void phase is smaller than $2 R$. A similar phenomenon occurs if the design variables vary smoothly between the solid and void phases: this also leads to a curve with a smaller slope and, consequently, a stronger erosion/dilation effect.

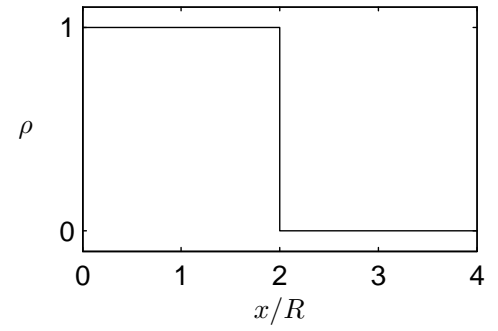

(a)

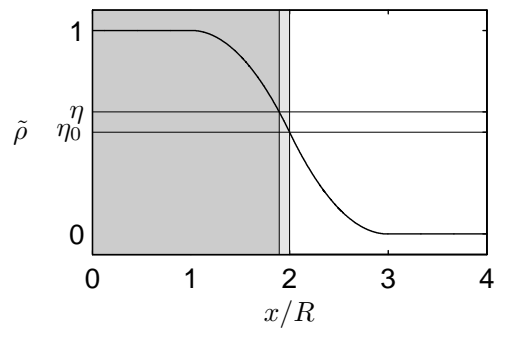

(c)

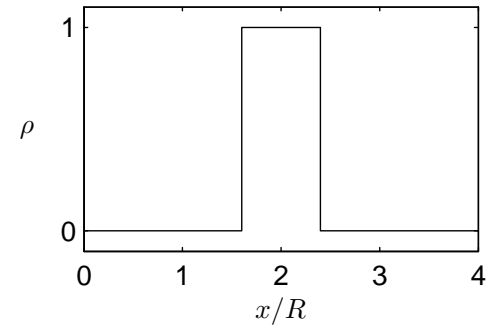

(b)

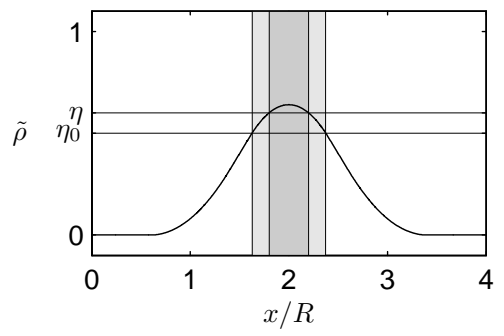

(d)

Figure 8: Design variable field $\boldsymbol{\rho}$ (top) and density filtered field $\tilde{\boldsymbol{\rho}}$ (bottom) at the transition from solid to void in 1D space. On the left hand side, the dimension of both the solid and the void phase is larger than 2 times the filter radius $R$. On the right hand side, a smaller solid phase is considered. The solid phase obtained by means of a Heaviside projection is visualized in the figures at the bottom: the gray zone (including both dark and light gray) is the solid phase obtained with a projection threshold $\eta_{0}=0.5$; the dark gray zone is what remains of the solid phase when the projection threshold is increased up to $\eta=0.6$.

Due to these phenomena, it is impossible to formulate a unique relationship between the projection threshold $\eta$ and the ratio $\varepsilon / R$. However, it is possible to determine a lower bound for $\varepsilon / R$ (which is exact for sharp boundaries between solid and void phases with dimensions larger than $2 R$ ). This lower bound is determined using figure $8 \mathrm{c}$, assuming that the reference design is obtained using $\eta_{0}=0.5$. The result is shown in figure 9 . Figure 9 allows for the selection of the 
appropriate values of $\eta$ (or the appropriate probability distribution $p_{\eta}(\eta)$ ) in order to model manufacturing errors with a given magnitude in a conservative way.

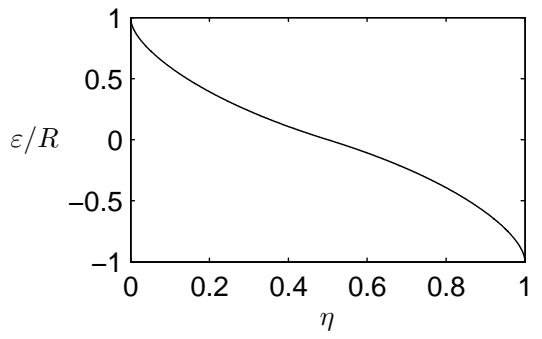

Figure 9: Relation between the Heaviside projection threshold $\eta$ and the magnitude $\varepsilon$ of the manufacturing error (normalized to the filter radius $R$ ) assuming that the reference design is obtained using $\eta_{0}=0.5$. A positive manufacturing error $\varepsilon$ is a dilation; a negative manufacturing error $\varepsilon$ is an erosion.

In the present study (where $R=8.4$ and $\eta$ varies between 0.4 and 0.6 ), the length scale $b$ for the intermediate design is equal to 5.31 for both the solid and the void phase (as long as the topology does not change due to the manufacturing errors; see reference [43]) and the erosion/dilation magnitude $\varepsilon$ varies between -0.91 and 0.91 .

\subsection{Non-uniform errors}

The strategy followed in the previous subsection can be modified to model non-uniform manufacturing errors (i.e. not constant over the entire design domain) by using a random field instead of a random variable to represent the projection threshold $\eta$. This approach is illustrated in figure 10: the spatially varying erosion/dilation effect is shown for three different realizations of the projection threshold.

Kolmogorov's probability theory allows for a rigorous definition of a random field (or a random process; a random field varies in space, a random process in time, but both are essentially identical) [12]. A random field is represented as a function $\eta(\mathbf{x}, \theta)$ of the spatial coordinates $\mathbf{x}$ and the elementary event $\theta$. The random field $\eta(\mathbf{x}, \theta)$ can be regarded as an infinite family of random variables $\eta_{\mathbf{x}_{i}}(\theta)=\eta\left(\mathbf{x}_{i}, \theta\right)$ at all points $\mathbf{x}_{i}$ in the domain where the random field is defined. A complete stochastic characterization of the random field $\eta(\mathbf{x}, \theta)$ requires the joint probability density functions of all combinations of the random variables $\eta_{\mathbf{x}_{i}}(\theta)$ to be specified. As this is practically impossible, the characterization of a random field is almost always incomplete.

It is common practice to characterize a random field by means of a marginal probability density function $p_{\eta_{\mathbf{x}}}(\eta)$, describing the behavior of the random field $\eta(\mathbf{x}, \theta)$ at a fixed position $\mathbf{x}$, and a covariance function $C_{\eta}\left(\mathbf{x}_{1}, \mathbf{x}_{2}\right)$, determining 

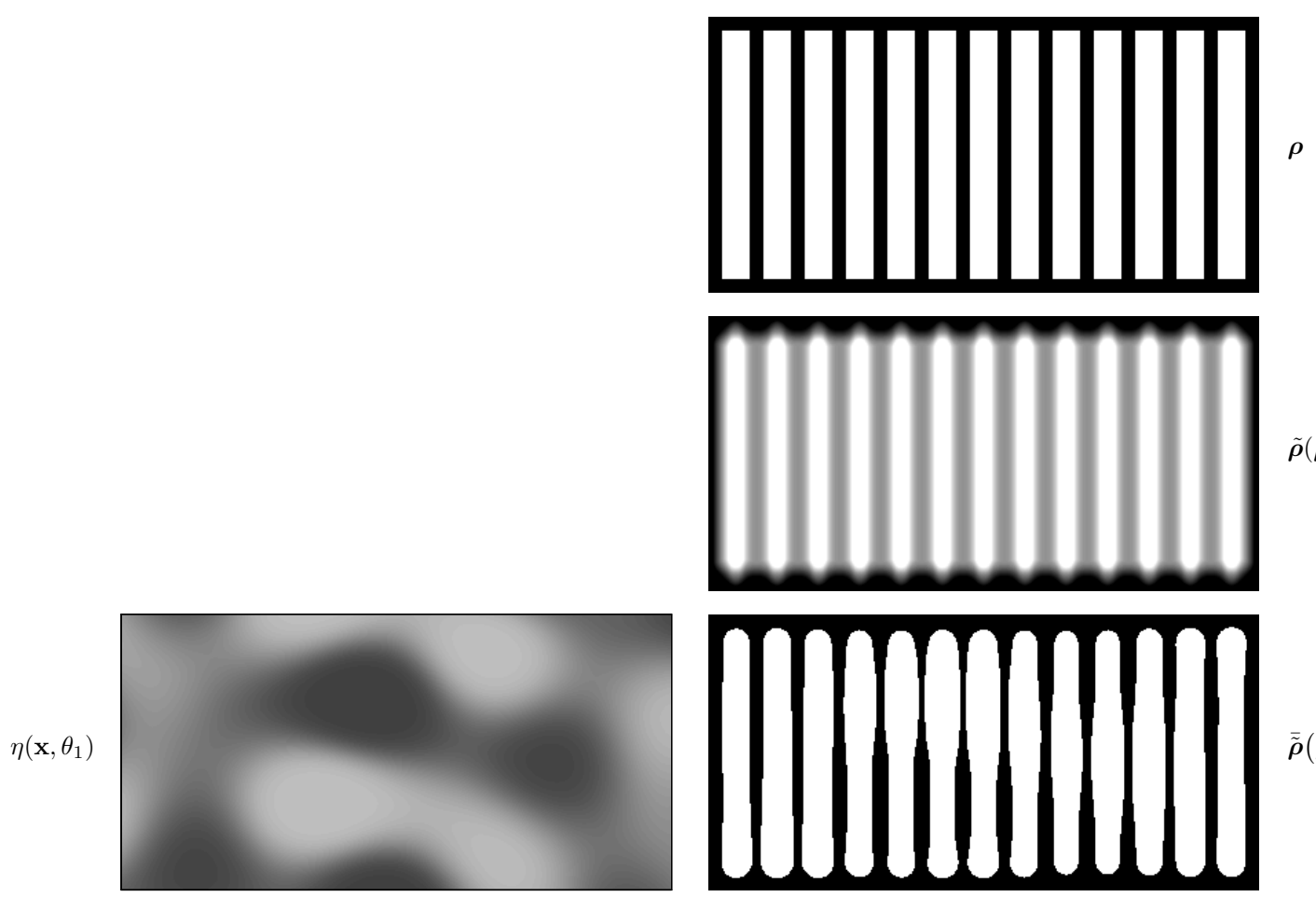

$\overline{\tilde{\boldsymbol{\rho}}}\left(\tilde{\boldsymbol{\rho}} \mid \eta\left(\mathbf{x}, \theta_{1}\right)\right)$
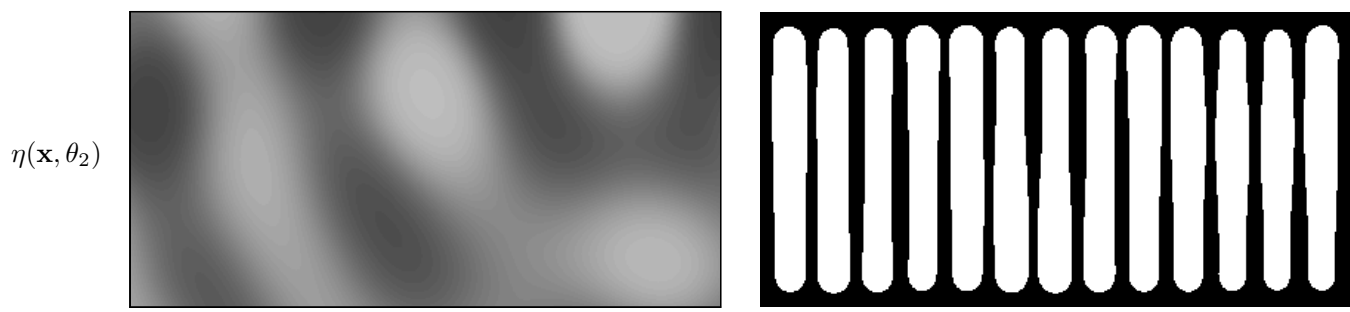

$\overline{\tilde{\boldsymbol{\rho}}}\left(\tilde{\boldsymbol{\rho}} \mid \eta\left(\mathbf{x}, \theta_{2}\right)\right)$
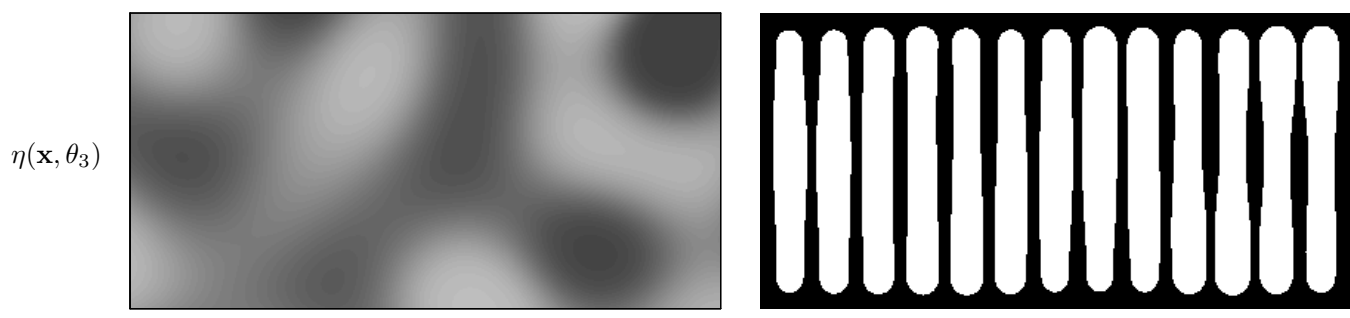

$\overline{\tilde{\boldsymbol{\rho}}}\left(\tilde{\boldsymbol{\rho}} \mid \eta\left(\mathbf{x}, \theta_{3}\right)\right)$

Figure 10: Non-uniform manufacturing errors modeled by means of a random Heaviside projection threshold $\eta(\mathbf{x}, \theta)$. The design variables $\boldsymbol{\rho}$, the intermediate variables $\tilde{\boldsymbol{\rho}}(\boldsymbol{\rho})$, and the element densities $\overline{\tilde{\boldsymbol{\rho}}}(\tilde{\boldsymbol{\rho}} \mid \eta(\mathbf{x}, \theta))$ obtained for three random realizations $\eta\left(\mathbf{x}, \theta_{1}\right), \eta\left(\mathbf{x}, \theta_{2}\right)$, and $\eta\left(\mathbf{x}, \theta_{3}\right)$ of the projection threshold are shown.

how the field varies in space. The covariance function $C_{\eta}\left(\mathbf{x}_{1}, \mathbf{x}_{2}\right)$ is defined as:

$$
C_{\eta}\left(\mathbf{x}_{1}, \mathbf{x}_{2}\right)=\mathbb{E}\left\{\left(\eta\left(\mathbf{x}_{1}, \theta\right)-\mathbb{E}\left\{\eta\left(\mathbf{x}_{1}, \theta\right)\right\}\right)\left(\eta\left(\mathbf{x}_{2}, \theta\right)-\mathbb{E}\left\{\eta\left(\mathbf{x}_{2}, \theta\right)\right\}\right)\right\}
$$


where $\mathbb{E}$ is the expectation operator. While the marginal probability density function $p_{\eta_{\mathrm{x}}}(\eta)$ determines the magnitude of the manufacturing errors (as discussed in the previous subsection), the covariance function $C_{\eta}\left(\mathbf{x}_{1}, \mathbf{x}_{2}\right)$ determines the variation of the manufacturing error in space. The choice of the marginal probability density function $p_{\eta_{\mathbf{x}}}(\eta)$ and the covariance function $C_{\eta}\left(\mathbf{x}_{1}, \mathbf{x}_{2}\right)$ depends on the properties of the production technique used.

Modeling a Gaussian random field (i.e. a random field with a Gaussian marginal probability density function) is less complicated than a non-Gaussian random field. Numerous methods for the discretization and simulation of Gaussian random fields have been presented in the literature. Among the most popular are series expansion methods such as the spectral representation method [35], the Karhunen-Loève expansion [46], and the Expansion Optimal Linear Estimation (EOLE) method [27].

As indicated in the previous subsection, the projection threshold $\eta(\mathbf{x}, \theta)$ cannot be modeled as a Gaussian random field. A random field with a uniform marginal probability density function $p_{\eta_{\mathbf{x}}}(\eta)$ between 0.4 and 0.6 is used instead. A non-Gaussian random field $\eta(\mathbf{x}, \theta)$ with a prescribed marginal probability density function $p_{\eta_{\mathbf{x}}}(\eta)$ and a prescribed covariance function $C_{\eta}\left(\mathbf{x}_{1}, \mathbf{x}_{2}\right)$ can be modeled as a translation process [16]:

$$
\eta(\mathbf{x}, \theta)=\gamma(\zeta(\mathbf{x}, \theta))=F_{\eta_{\mathbf{x}}}^{-1}\left(F_{\zeta}(\zeta(\mathbf{x}, \theta))\right)
$$

where $\zeta(\mathbf{x}, \theta)$ is a standard Gaussian random field and $\gamma$ is a memoryless transformation defined in a similar way as in equation (12), using the marginal cumulative distribution function $F_{\eta_{\mathbf{x}}}(\eta)$ of the random field $\eta(\mathbf{x}, \theta)$ and the standard Gaussian cumulative distribution function $F_{\zeta}(\zeta)$. Grigoriu [16] proposes a procedure similar to the Nataf transformation [28] to determine the covariance function $C_{\zeta}\left(\mathbf{x}_{1}, \mathbf{x}_{2}\right)$ of the underlying Gaussian random field $\zeta(\mathbf{x}, \theta)$ so that the memoryless transformation $\gamma$ leads to a random field $\eta(\mathbf{x}, \theta)$ with the prescribed covariance function $C_{\eta}\left(\mathbf{x}_{1}, \mathbf{x}_{2}\right)$. However, this procedure does not always lead to a valid solution (i.e. to a covariance function $C_{\zeta}\left(\mathbf{x}_{1}, \mathbf{x}_{2}\right)$ that is positive definite). In order to avoid such problems here, the covariance function $C_{\zeta}\left(\mathbf{x}_{1}, \mathbf{x}_{2}\right)$ is chosen directly instead of choosing a covariance function $C_{\eta}\left(\mathbf{x}_{1}, \mathbf{x}_{2}\right)$ and attempting to determine the corresponding covariance function $C_{\zeta}\left(\mathbf{x}_{1}, \mathbf{x}_{2}\right)$.

A covariance function $C_{\zeta}\left(\mathbf{x}_{1}, \mathbf{x}_{2}\right)$ must be positive definite in order to be valid [46]. For multidimensional random fields, a covariance function depending on the Euclidean distance $\left\|\mathbf{x}_{2}-\mathbf{x}_{1}\right\|$ is often used. This approach allows for the use of a 1D covariance function in a higher dimensional space. However, it is important to realize that not all covariance functions that are positive definite in 1D space remain positive definite in higher dimensions, as explained by Weber and Talkner [44].

In the present study, a squared exponential covariance function is used:

$$
C_{\zeta}\left(\mathbf{x}_{1}, \mathbf{x}_{2}\right)=\exp \left(-\frac{\left\|\mathbf{x}_{2}-\mathbf{x}_{1}\right\|^{2}}{l_{\mathrm{c}}^{2}}\right)
$$

where $l_{\mathrm{c}}$ is the correlation length. The correlation length determines the scale 
of variation of the random field $\zeta(\mathbf{x}, \theta)$ : a random field with a small correlation length will exhibit more fine scale variations than a random field with a large correlation length. A correlation length $l_{\mathrm{c}}=0.3 \mathrm{~L}$ is used. The covariance function $C_{\zeta}\left(\mathbf{x}_{1}, \mathbf{x}_{2}\right)$ is visualized in figure 11 .

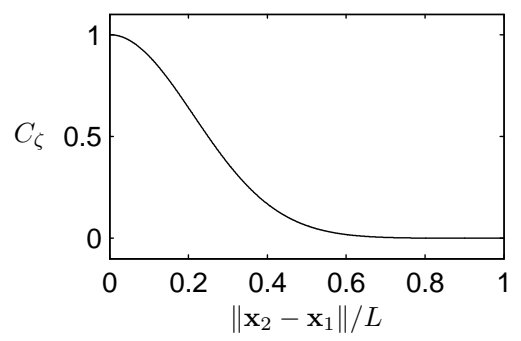

Figure 11: Covariance function $C_{\zeta}\left(\mathbf{x}_{1}, \mathbf{x}_{2}\right)$ of the underlying standard Gaussian random field $\zeta(\mathbf{x}, \theta)$.

The squared exponential covariance function has certain advantages over the exponential covariance function $\exp \left(-\left\|\mathbf{x}_{2}-\mathbf{x}_{1}\right\| / l_{\mathrm{c}}\right)$, which is also frequently used: the former represents a differentiable random field [46], which is more realistic for most physical properties, and can be modeled more accurately due to its smoothness [40].

The underlying standard Gaussian random field $\zeta(\mathbf{x}, \theta)$ is modeled by means of the EOLE method [27]. The EOLE method consists of two steps. In the first step, a number $N$ of nodal points $\mathbf{x}_{1}, \ldots, \mathbf{x}_{N}$ in the domain of the random field is selected. The values of the random field at these points are collected in a vector $\mathbf{Z}=\left\{\zeta\left(\mathbf{x}_{1}, \theta\right), \ldots, \zeta\left(\mathbf{x}_{N}, \theta\right)\right\}^{\mathrm{T}}$. The value at any other location $\mathbf{x}$ is approximated by means of the Optimal Linear Estimation (OLE) method:

$$
\zeta(\mathbf{x}, \theta) \approx \mathbf{C}_{\mathbf{Z} \zeta}^{\mathrm{T}}(\mathbf{x}) \mathbf{C}_{\mathbf{Z}}^{-1} \mathbf{Z}(\theta)
$$

where $\mathbf{C}_{\mathbf{Z}}$ is the correlation matrix of the random vector $\mathbf{Z}(\theta)$ :

$$
\mathbf{C}_{\mathbf{Z}}=\left[\begin{array}{ccc}
C_{\zeta}\left(\mathbf{x}_{1}, \mathbf{x}_{1}\right) & \ldots & C_{\zeta}\left(\mathbf{x}_{1}, \mathbf{x}_{N}\right) \\
\vdots & \ddots & \vdots \\
C_{\zeta}\left(\mathbf{x}_{N}, \mathbf{x}_{1}\right) & \ldots & C_{\zeta}\left(\mathbf{x}_{N}, \mathbf{x}_{N}\right)
\end{array}\right]
$$

and $\mathbf{C}_{\mathbf{Z} \zeta}(\mathbf{x})$ is the cross-correlation matrix of the vector $\mathbf{Z}(\theta)$ and the scalar $\zeta(\mathbf{x}, \theta)$ :

$$
\mathbf{C}_{\mathbf{Z} \zeta}(\mathbf{x})=\left\{\begin{array}{c}
C_{\zeta}\left(\mathbf{x}_{1}, \mathbf{x}\right) \\
\vdots \\
C_{\zeta}\left(\mathbf{x}_{N}, \mathbf{x}\right)
\end{array}\right\}
$$

In the second step, the components of the random vector $\mathbf{Z}(\theta)$ are decorrelated by means of principal component analysis [18]:

$$
\mathbf{Z}(\theta)=\sum_{k=1}^{N} \xi_{k}(\theta) \sqrt{\lambda}_{k} \mathbf{v}_{k}
$$


where $\xi_{k}(\theta)$ are uncorrelated random variables with unit variance and $\lambda_{k}$ and $\mathbf{v}_{k}$ are the eigenvalues and eigenvectors of the covariance matrix $\mathbf{C}_{\mathbf{Z}}$. Since the vector $\mathbf{Z}(\theta)$ follows a Gaussian distribution, the random variables $\xi_{k}(\theta)$ are independent standard Gaussian variables.

Combining equations (16) and (19) gives the EOLE approximation of the random field $\zeta(\mathbf{x}, \theta)$ :

$$
\zeta(\mathbf{x}, \theta) \approx \sum_{k=1}^{N} \xi_{k}(\theta) \frac{1}{\sqrt{\lambda}_{k}} \mathbf{v}_{k}^{\mathrm{T}} \mathbf{C}_{\mathbf{Z} \zeta}(\mathbf{x})
$$

where use is made of the properties $\mathbf{C}_{\mathbf{Z}}^{-1}=\sum_{j=1}^{N} \mathbf{v}_{j} \lambda_{j}^{-1} \mathbf{v}_{j}^{\mathrm{T}}$ and $\mathbf{v}_{j}^{\mathrm{T}} \mathbf{v}_{k}=\delta_{j k}$, with $\delta_{j k}$ the Kronecker Delta. The series in equation (20) can be truncated after $M \leq N$ terms in order to reduce the dimensionality of the problem (provided that the eigenvalues $\lambda_{k}$ are sorted in decreasing order).

Equation (20) is reformulated as:

$$
\zeta(\mathbf{x}, \theta) \approx \sum_{k=1}^{M} \xi_{k}(\theta) \phi_{k}(\mathbf{x})
$$

where $\phi_{k}(\mathbf{x})=\frac{1}{\sqrt{\lambda_{k}}} \mathbf{v}_{k}^{\mathrm{T}} \mathbf{C}_{\mathbf{z} \zeta}(\mathbf{x})$. This equation makes clear that the EOLE expansion of the (standard Gaussian) random field $\zeta(\mathbf{x}, \theta)$ is a linear combination of deterministic functions $\phi_{k}(\mathbf{x})$ with (independent standard Gaussian) random coefficients $\xi_{k}(\theta)$, similar to the Karhunen-Loève expansion.

The Karhunen-Loève expansion requires the solution of an integral eigenvalue problem. For a random field with a squared exponential covariance function, this problem cannot be solved analytically, and only an approximate solution based on interpolation functions can be obtained (e.g. by means of the Galerkin type procedure proposed by Ghanem and Spanos [15]). Li and Der Kiureghian [27] demonstrated that the EOLE expansion is more efficient than the Karhunen-Loève expansion in such cases.

The locations of the nodal points used for the EOLE expansion are indicated in figure 12. A regular EOLE mesh with equally spaced nodes is used, which is possible in this case due to the square shape of the design domain. However, the use of a regular mesh or equally spaced nodes is not a requirement, which means that the EOLE method is equally applicable in cases where the design domain has a more complex shape. The number $N$ of nodal points is equal to 100 ; this number is chosen so that the size of each element in the EOLE mesh is smaller than $l_{\mathrm{c}} / 3$, as recommended by Sudret and Der Kiureghian [40] for random fields with a squared exponential covariance function.

Figure 13 shows the first ten functions $\phi_{k}(\mathbf{x})$ in the EOLE expansion. These functions are approximations to the modes in a Karhunen-Loève expansion and can therefore be referred to as EOLE modes. It can be observed that the lowest order modes represent the coarse scale variations in the random field $\zeta(\mathbf{x}, \theta)$, while the higher order modes represent the fine scale variations. The magnitude of the modes decreases with the mode number. As a result, the importance 


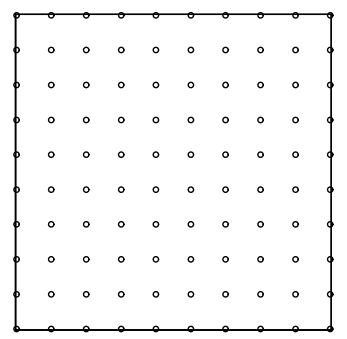

Figure 12: Locations of the EOLE nodal points in the design domain.

of the highest order modes in the EOLE expansion is limited, which justifies a truncation of the expansion after a limited number of terms. In the present analysis no truncation is performed, however, and all modes are retained in the expansion (i.e. $M=N=100$ ).
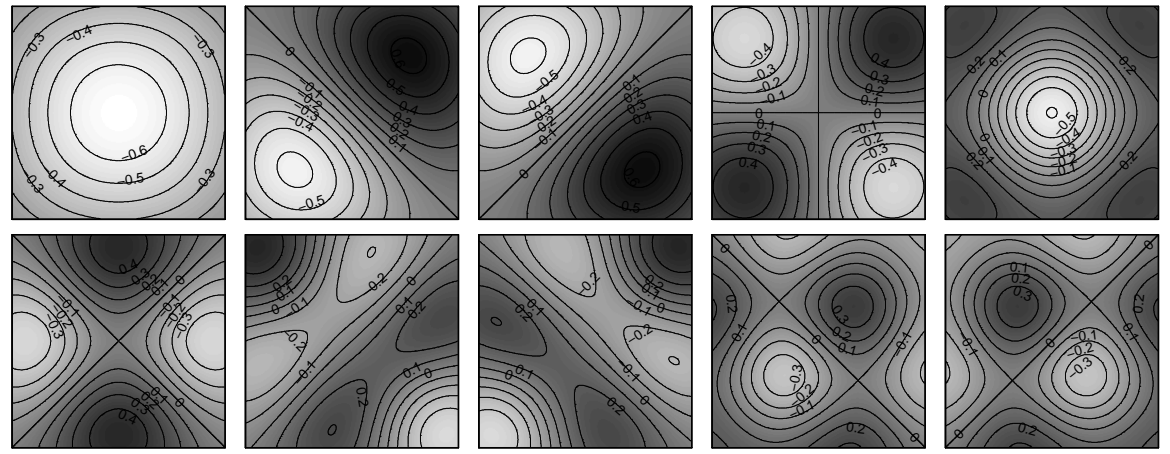

Figure 13: First ten modes $\phi_{k}(\mathbf{x})$ in the EOLE expansion of the underlying Gaussian process $\zeta(\mathbf{x}, \theta)$.

The random Heaviside projection threshold $\eta(\mathbf{x}, \theta)$ is now simulated as follows: a random number generator is used to generate realizations of the standard Gaussian variables $\xi_{k}(\theta)$. These realizations are introduced in equation (21) to obtain a realization of the underlying Gaussian field $\zeta(\mathbf{x}, \theta)$. This realization is finally transformed into a realization of the projection threshold $\eta(\mathbf{x}, \theta)$ by means of the memoryless transformation in equation (14).

Five realizations of the underlying Gaussian random field $\zeta(\mathbf{x}, \theta)$ as well as the corresponding realizations of the random projection threshold $\eta(\mathbf{x}, \theta)$ are shown in figure 14. It can be observed that the realizations of both random fields $\zeta(\mathbf{x}, \theta)$ and $\eta(\mathbf{x}, \theta)$ vary in space in a similar way, which suggests that the impact of prescribing the covariance function $C_{\zeta}\left(\mathbf{x}_{1}, \mathbf{x}_{2}\right)$ of the underlying Gaussian random field $\zeta(\mathbf{x}, \theta)$ instead of the covariance function $C_{\eta}\left(\mathbf{x}_{1}, \mathbf{x}_{2}\right)$ of the random field of interest $\eta(\mathbf{x}, \theta)$ is relatively low.

Figure 15 shows the effect of an non-uniform manufacturing error for the example structure with a circular hole introduced in subsection 3.1. As can be 

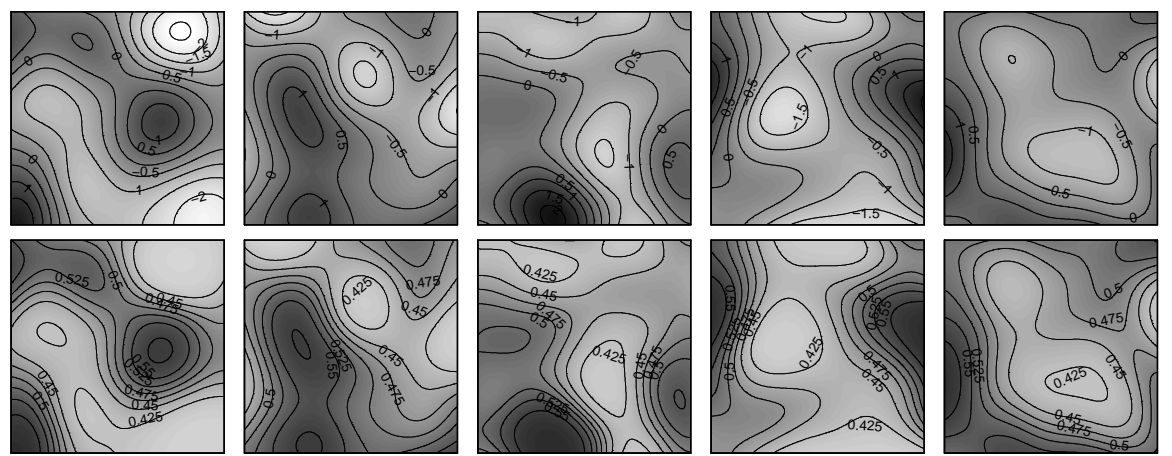

Figure 14: Five realizations of the underlying Gaussian random field $\zeta(\mathbf{x}, \theta)$ (top) and the random Heaviside projection threshold $\eta(\mathbf{x}, \theta)$ (bottom).

observed, the result is a structure with a non-circular hole. The magnitude of the manufacturing error $\varepsilon$ is determined by the radius $R$ of the density filter and the variation of the projection threshold $\eta(\mathbf{x}, \theta)$, in the same way as for uniform manufacturing errors.

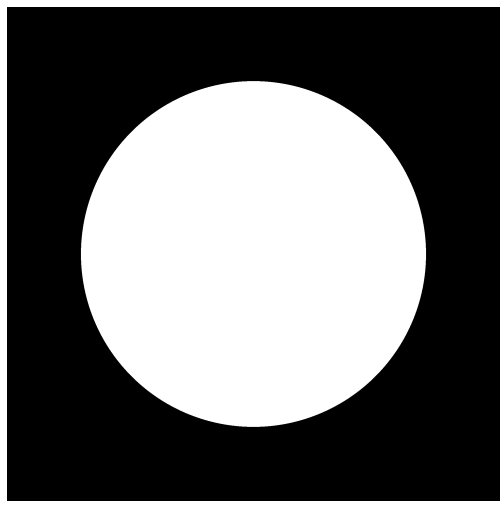

(a)

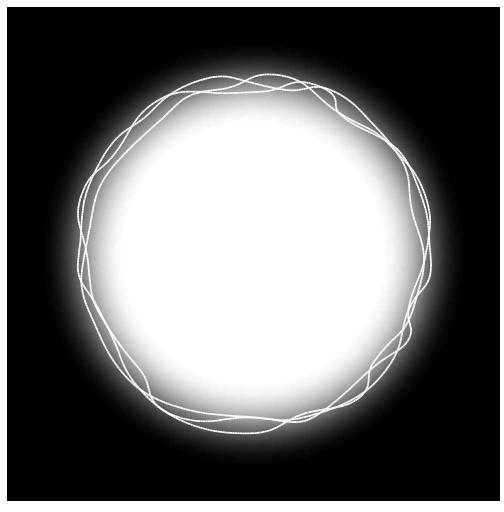

(b)

Figure 15: (a) Design variables $\boldsymbol{\rho}$ for a square structure with a circular hole and (b) corresponding filtered variables $\tilde{\boldsymbol{\rho}}(\boldsymbol{\rho})$. The element densities $\overline{\tilde{\boldsymbol{\rho}}}(\tilde{\boldsymbol{\rho}} \mid \eta(\mathbf{x}, \theta))$ are computed for three realizations $\eta\left(\mathbf{x}, \theta_{1}\right), \eta\left(\mathbf{x}, \theta_{2}\right)$, and $\eta\left(\mathbf{x}, \theta_{3}\right)$ of the projection threshold. For each realization, the boundary between the solid and the void phase is indicated by means of a line.

\section{Robust topology optimization}

\subsection{Formulation of the optimization problem}

We now have expressed the manufacturing uncertainties in terms of a standard Gaussian vector $\boldsymbol{\xi}(\theta)$. This vector $\boldsymbol{\xi}(\theta)$ contains only a single random variable $\xi(\theta)$ in the case of uniform errors and $M$ random variables $\xi_{k}(\theta)$ in the 
case of non-uniform errors. The response $f=\mathrm{l}^{\mathrm{T}} \mathbf{u}$ of the structure depends both on the design variables $\boldsymbol{\rho}$ and on the uncertain parameters $\boldsymbol{\xi}(\theta)$ and is therefore denoted as $f(\boldsymbol{\rho}, \boldsymbol{\xi}(\theta))$. Similarly, the volume constraint function is denoted as $g(\boldsymbol{\rho}, \boldsymbol{\xi}(\theta))$.

Robust design optimization implies that we aim for a low value of the response that is not too sensitive to the uncertain parameters $\boldsymbol{\xi}(\theta)$. Several approaches to the mathematical formulation of this objective have been followed in the literature; an extensive overview is given in reference [6]. In the present study, the robust optimization problem is formulated as follows:

$$
\begin{aligned}
\min _{\boldsymbol{\rho}} & : F(\boldsymbol{\rho})=m_{f}(\boldsymbol{\rho})+\kappa \sigma_{f}(\boldsymbol{\rho}) \\
\text { s.t. } & : \mathbf{K}(\boldsymbol{\rho}, \boldsymbol{\xi}) \mathbf{u}(\boldsymbol{\rho}, \boldsymbol{\xi})=\mathbf{f} \\
& : G(\boldsymbol{\rho})=m_{g}(\boldsymbol{\rho}) \leq V^{*} \\
& : \mathbf{0} \leq \boldsymbol{\rho} \leq \mathbf{1}
\end{aligned}
$$

where $m_{f}(\boldsymbol{\rho})$ and $\sigma_{f}(\boldsymbol{\rho})$ are the mean value and the standard deviation of the response $f(\boldsymbol{\rho}, \boldsymbol{\xi}(\theta))$ :

$$
\begin{aligned}
& m_{f}(\boldsymbol{\rho})=\mathbb{E}\{f(\boldsymbol{\rho}, \boldsymbol{\xi}(\theta))\} \\
& \sigma_{f}(\boldsymbol{\rho})=\sqrt{\mathbb{E}\left\{[f(\boldsymbol{\rho}, \boldsymbol{\xi}(\theta))]^{2}-\left[\mathbb{E}\{f(\boldsymbol{\rho}, \xi(\theta)\}]^{2}\right\}\right.}
\end{aligned}
$$

The factor $\kappa$ in equation (22) is a weighting factor which is chosen equal to 1 in the present study. It should be noted that this choice has an influence on the result of the optimization: increasing $\kappa$ leads to a design with a lower sensitivity to manufacturing errors but a worse mean performance. The volume constraint $G(\boldsymbol{\rho})$ is formulated in terms of the mean value $m_{g}(\boldsymbol{\rho})$ of the constraint function $g(\boldsymbol{\rho}, \boldsymbol{\xi}(\theta))$ :

$$
m_{g}(\boldsymbol{\rho})=\mathbb{E}\{g(\boldsymbol{\rho}, \boldsymbol{\xi}(\theta))\}
$$

From a designer's point of view, it may be more logical to express the volume constraint in terms of the reference or blueprint design, i.e. the design obtained with a projection threshold $\eta_{0}=0.5$. However, this would lead to an ill-conditioned optimization problem and dilated designs with unrealistically thick bars, as explained in reference [37].

Owing to the linearity of the expectation operator $\mathbb{E}$, the sensitivity $\frac{\partial m_{f}}{\partial \rho_{k}}$ of the mean response $m_{f}$ with respect to the design variables $\rho_{k}$ is simply obtained as the mean sensitivity of the random response $f(\boldsymbol{\rho}, \boldsymbol{\xi}(\theta))$ :

$$
\frac{\partial m_{f}}{\partial \rho_{k}}=\mathbb{E}\left\{\frac{\partial f}{\partial \rho_{k}}\right\}
$$

The sensitivity $\frac{\partial m_{g}}{\partial \rho_{k}}$ of the mean volume constraint $m_{g}$ with respect to the design variables $\rho_{k}$ is obtained in a similar way. Using the chain rule, the sensitivity 
$\frac{\partial \sigma_{f}}{\partial \rho_{k}}$ of the standard deviation $\sigma_{f}$ of the response with respect to the design variables $\rho_{k}$ is determined as:

$$
\frac{\partial \sigma_{f}}{\partial \rho_{k}}=\frac{1}{\sigma_{f}} \mathbb{E}\left\{\left(f-m_{f}\right) \frac{\partial f}{\partial \rho_{k}}\right\}
$$

The robust optimization problem is solved with MMA, using the same parameters, the same continuation scheme, and the same number of iterations as in the deterministic case. In each optimization iteration, the statistical moments $m_{f}(\boldsymbol{\rho}), \sigma_{f}(\boldsymbol{\rho})$, and $m_{g}(\boldsymbol{\rho})$ and their sensitivities $\frac{\partial m_{f}}{\partial \rho_{k}}, \frac{\partial \sigma_{f}}{\partial \rho_{k}}$, and $\frac{\partial m_{g}}{\partial \rho_{k}}$ are estimated by means of a Monte Carlo method [30].

In each Monte Carlo simulation, 100 realizations $\boldsymbol{\xi}\left(\theta_{i}\right)$ of the uncertain parameters are considered. The same realizations are used throughout the entire iteration history in order to ensure smooth convergence of the optimization scheme. The number of realizations is relatively low, so that the computational cost of the optimization scheme remains reasonable. For the final design, a more elaborate Monte Carlo simulation is performed in order to verify the accuracy of the estimates of the mean value and the standard deviation of the response $f(\boldsymbol{\rho}, \boldsymbol{\xi}(\theta))$. In the following, the estimates of these statistics obtained from the simulation with 100 realizations (which are used for the optimization) are denoted as $\hat{m}_{f}$ and $\hat{\sigma}_{f}$, while the estimates obtained from the simulation with 10000 realizations (which serve as reference values) are denoted as $m_{f}$ and $\sigma_{f}$.

As 100 designs are analyzed in each iteration of the optimization scheme, the computational cost is 100 times as high as in the deterministic case. However, the fact that all designs are relatively similar allows for the use of approximate reanalysis techniques $[1,2]$, so that the cost can at least partially be alleviated. A preliminary study has shown that a speedup by a factor of 5 is possible. For low dimensional problems (in stochastic space; i.e. problems where the number of modes in the EOLE expansion is limited), alternative probabilistic techniques such as the perturbation method [15, 22], polynomial chaos expansion [15], and sparse grid quadrature [14] have proven to be more efficient than a Monte Carlo method [26].

\subsection{Compliant mechanism design}

Figure 16 shows the optimized compliant mechanism design obtained with a robust approach considering uniform and non-uniform manufacturing errors. The figure shows the design variables $\boldsymbol{\rho}$, the filtered variables $\tilde{\boldsymbol{\rho}}(\boldsymbol{\rho})$, and the element densities $\overline{\tilde{\boldsymbol{\rho}}}\left(\tilde{\boldsymbol{\rho}} \mid \eta_{0}\right)$ for the reference design (obtained with a projection threshold $\eta_{0}=0.5$ ). The designs obtained for uniform and non-uniform manufacturing errors are very similar. Compared with the design obtained by deterministic optimization (figure 2), the most obvious difference is the absence of narrow hinges. As a consequence, the risk of failure due to over-etching is eliminated.

In the case of uniform manufacturing errors, the performance of the reference design is $f_{0}=-1.851$, which is slightly worse than the value $f_{0}=-2.023$ for the deterministic design. The mean response $\hat{m}_{f}=-1.818$ is very close to 


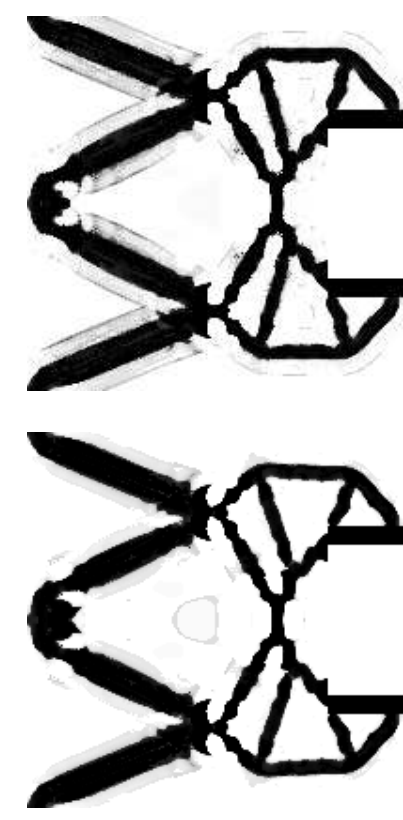

(a) Design variables $\rho$.

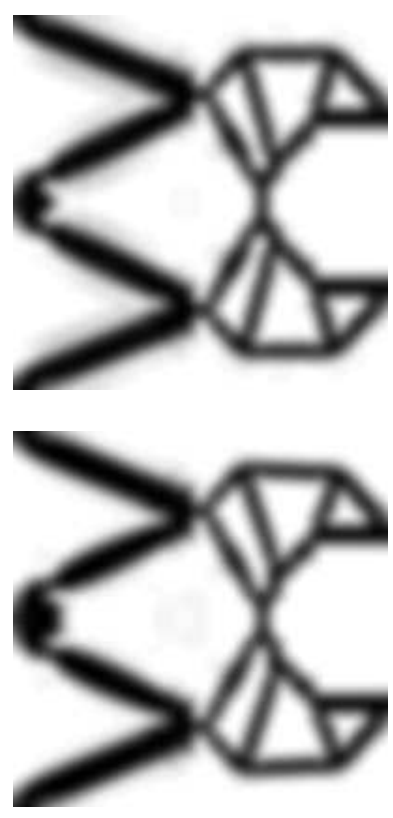

(b) Filtered variables $\tilde{\boldsymbol{\rho}}(\boldsymbol{\rho})$.

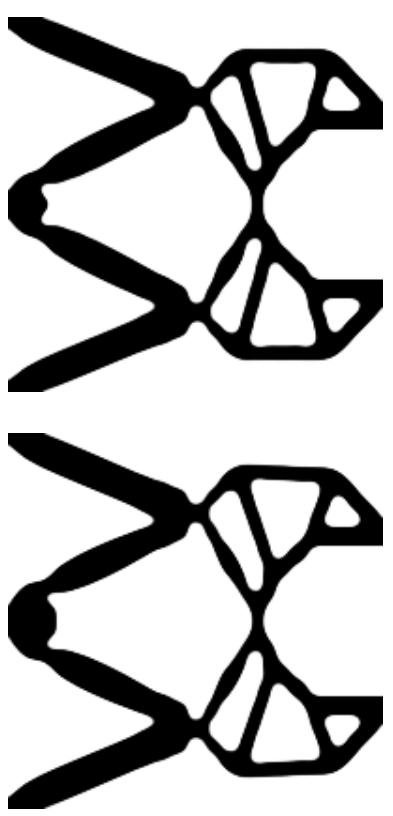

(c) Element densities $\overline{\tilde{\boldsymbol{\rho}}}\left(\tilde{\boldsymbol{\rho}} \mid \eta_{0}\right)$.

Figure 16: Optimized design for a compliant gripper mechanism obtained by robust optimization considering uniform (top) and non-uniform (bottom) manufacturing errors.

the response $f_{0}$ of the reference design, and the standard deviation $\hat{\sigma}_{f}=0.035$ is very small, which means that a high degree of robustness is achieved: the optimized design is almost insensitive with respect to uniform manufacturing errors. The estimates $\hat{m}_{f}$ and $\hat{\sigma}_{f}$ of the response statistics are very close to the reference values $m_{f}=-1.817$ and $\sigma_{f}=0.035$ obtained by means of a more elaborate Monte Carlo simulation. It can therefore be concluded that using 100 samples in the optimization process is sufficient for this particular problem.

Very similar results are obtained in the case of non-uniform manufacturing errors: the response of the reference design is $f_{0}=-1.866$, the estimates of the mean response and standard deviation are $\hat{m}_{f}=-1.825$ and $\hat{\sigma}_{f}=0.037$, and the reference values obtained with a more thorough Monte Carlo simulation are $m_{f}=-1.821$ and $\sigma_{f}=0.036$.

The impact of uniform and non-uniform manufacturing errors on the three designs (the design obtained by deterministic optimization and both designs obtained by robust optimization) is studied by means of additional Monte Carlo simulations. Figure 17 shows the effect for three realizations of the random manufacturing error. The target length scale $b$ for the reference design and the target range of the manufacturing error magnitude $\varepsilon$ are also indicated in the figure. It can be observed that the magnitude of the errors in the design corresponds well with the target. 


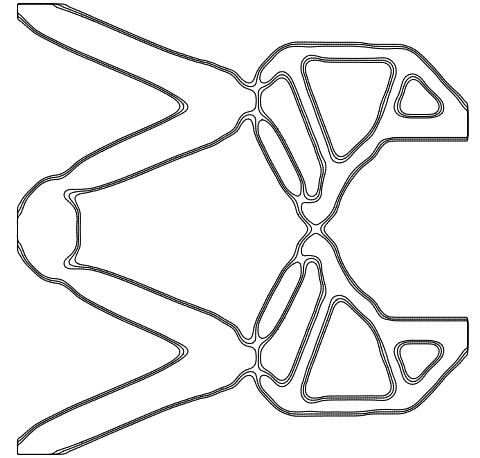

(a) $m_{f}=-1.310, \sigma_{f}=0.824$.

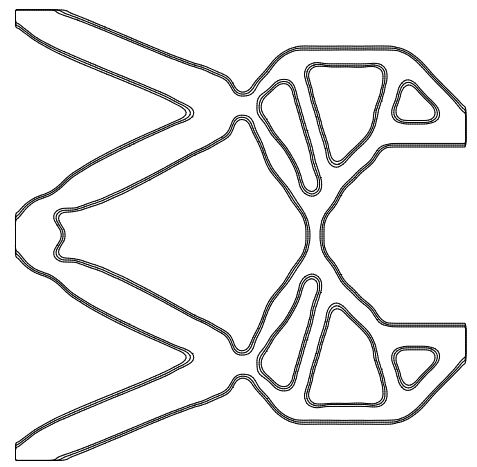

(c) $m_{f}=-1.817, \sigma_{f}=0.035$.

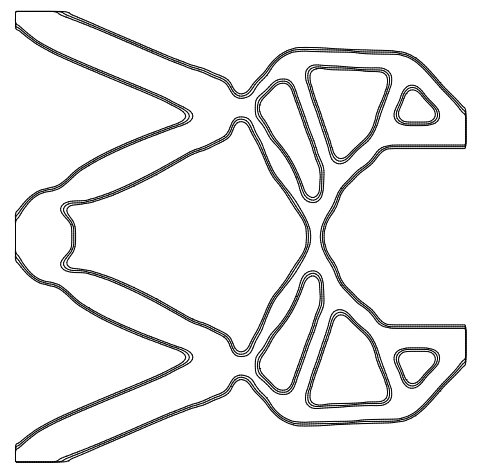

(e) $m_{f}=-1.822, \sigma_{f}=0.050$.

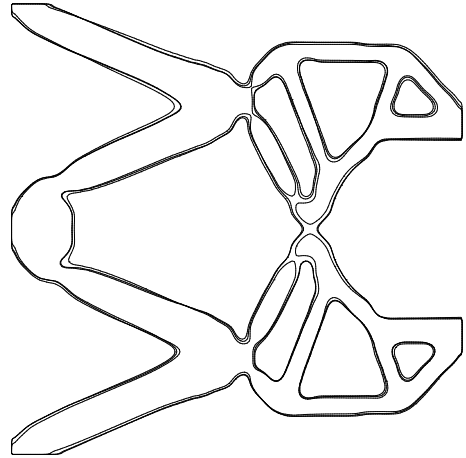

(b) $m_{f}=-0.846, \sigma_{f}=0.843$.

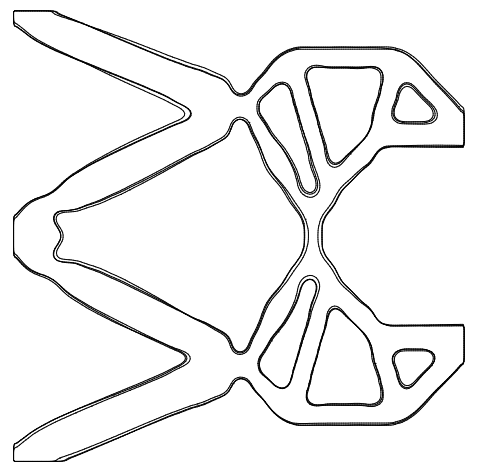

(d) $m_{f}=-1.815, \sigma_{f}=0.035$.

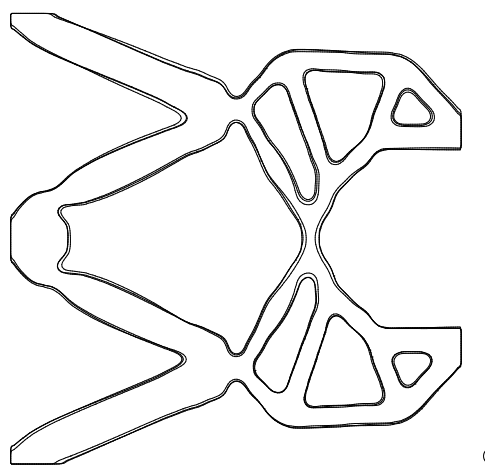

(f) $m_{f}=-1.821, \sigma_{f}=0.036$.

Figure 17: The effect of uniform (left) and non-uniform (right) manufacturing errors on the optimized gripper mechanism design obtained assuming no manufacturing errors (top), uniform manufacturing errors (middle), and non-uniform manufacturing errors (bottom). The top circle in the bottom right corner represents the target length scale $b$ (in the reference design) of both solid and void regions. The difference between the top circle and the bottom circles represents the target magnitude $\varepsilon$ of the erosion/dilation errors. 
The deterministic design (figures $17 \mathrm{a}$ and $17 \mathrm{~b}$ ) is very sensitive to manufacturing errors due to the occurrence of narrow hinges. The mechanism remains intact for some realizations of the manufacturing error, but it fails for others. As a consequence, the mean performance of the mechanism degrades ( $m_{f}=-1.310$ for uniform errors; $m_{f}=-0.846$ for non-uniform errors) and the standard deviation increases strongly $\left(\sigma_{f}=0.824\right.$ for uniform errors; $\sigma_{f}=0.843$ for non-uniform errors).

The designs obtained assuming uniform manufacturing errors (figures $17 \mathrm{c}$ and $17 \mathrm{~d}$ ) and assuming non-uniform manufacturing errors (figures 17e and 17f) are very similar. Both designs remain intact for both types of the manufacturing error. Moreover, the mean value $m_{f}$ and the standard deviation $\sigma_{f}$ of the response (specified in the figure) are almost identical for both designs and both types of error. It can therefore be concluded that, for this particular problem, the design obtained assuming uniform manufacturing errors is equally robust with respect to non-uniform errors, and vice versa.

\subsection{Heat sink design}

Figure 18 shows the optimized heat sink design obtained by means of a robust approach assuming uniform and non-uniform manufacturing errors. The clearest difference with the design obtained by deterministic optimization (figure 4) is the presence of a length scale in both the solid and the void phase.
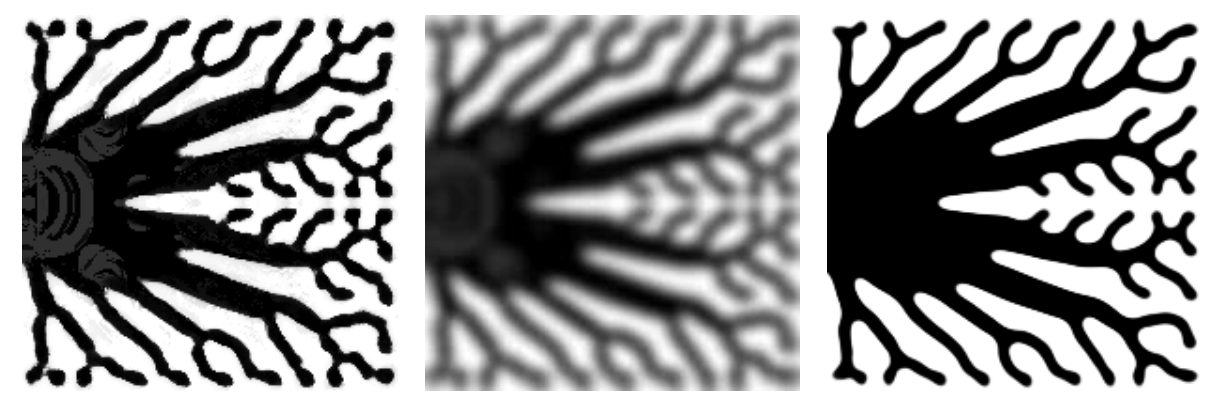

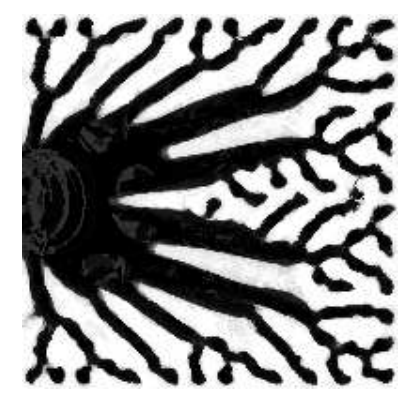

(a) Design variables $\boldsymbol{\rho}$.

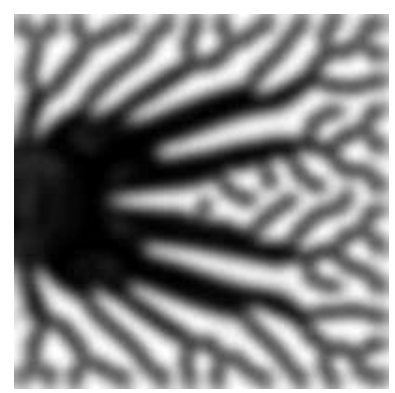

(b) Filtered variables $\tilde{\boldsymbol{\rho}}(\boldsymbol{\rho})$.

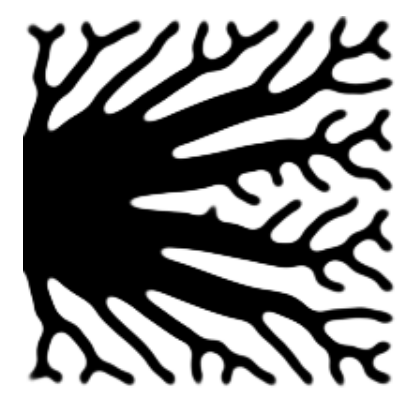

(c) Element densities $\overline{\tilde{\boldsymbol{\rho}}}\left(\tilde{\boldsymbol{\rho}} \mid \eta_{0}\right)$.

Figure 18: Optimized design for a heat sink obtained by robust optimization considering uniform (top) and non-uniform (bottom) manufacturing errors. 
Both designs in figure 18 are quite similar, except that the one obtained for uniform errors is symmetric, while the other one is asymmetric. The asymmetry may be due to the fact that only a limited number of (asymmetric) manufacturing errors is considered in the optimization. However, this is probably not the only reason, as the deterministic optimization scheme also leads to an asymmetric design (figure 4). A possible explanation is that the formation of an asymmetric pattern allows for a more even distribution of conductive material along the axis of symmetry of the design domain.

In the case where uniform errors are considered, the response of the reference design is $f_{0}=1.277$, which is slightly worse than the value $f_{0}=1.179$ obtained in the deterministic case. The mean response and the standard deviation are estimated as $\hat{m}_{f}=1.303$ and $\hat{\sigma}_{f}=0.099$. These estimates are very close to the reference values $m_{f}=1.299$ and $\sigma_{f}=0.098$ obtained with a more thorough Monte Carlo simulation, which suggests that using 100 samples for the optimization is sufficient.

In the case of non-uniform manufacturing errors, the response of the reference design is $f_{0}=1.268$, the mean response and the standard deviation are estimated as $\hat{m}_{f}=1.291$ and $\hat{\sigma}_{f}=0.042$, and the reference values are $m_{f}=1.296$ and $\sigma_{f}=0.050$. These results are similar to the values obtained for uniform errors, except for the standard deviation $\sigma_{f}$, which is two times smaller. This can be explained by the monotonic behavior of the objective function with respect to the element densities: a dilation (increasing element densities) will always lead to a larger heat transfer, while an erosion (decreasing element densities) will always lead to a smaller heat transfer. In the case of non-uniform manufacturing errors, dilations occur in some regions of the design domain, while erosions occur in other regions. The total amount of material does not change as strongly as in the case of uniform manufacturing errors, and the variation of the objective function remains limited.

As in the previous subsection, the effect of the two different types of manufacturing errors on the three designs is studied by means of additional Monte Carlo simulations. Figure 19 shows this effect for three realizations of the manufacturing error. The target length scale in the intermediate design and the target range of erosion/dilation errors are also indicated.

For the design obtained by deterministic optimization (figures 19a and 19b), the manufacturing errors are much larger than intended. This is due to the absence of a length scale in the design: as explained in subsection 3.1, the relationship between the Heaviside projection threshold $\eta$ and the magnitude of the manufacturing errors $\varepsilon$ shown in figure 9 only holds for sharp boundaries between solid and void phases with dimensions larger than $2 R$. In this case, the dimensions of the phases are much smaller, and this results in a stronger (unrealistically strong) erosion/dilation effect. As a consequence, the statistics $m_{f}$ and $\sigma_{f}$ given in figures $19 \mathrm{a}$ and $19 \mathrm{~b}$ are meaningless. Nevertheless, it is clear that a design without a length scale cannot be robust with respect to (uniform or non-uniform) manufacturing errors.

For the robust designs (figures 19c to 19f), the magnitude of the manufacturing errors agrees with the target. The effect of uniform manufacting errors on 


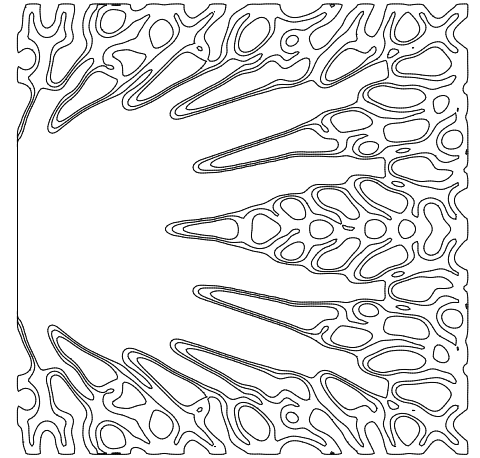

(a) $m_{f}=2.142, \sigma_{f}=1.641$.

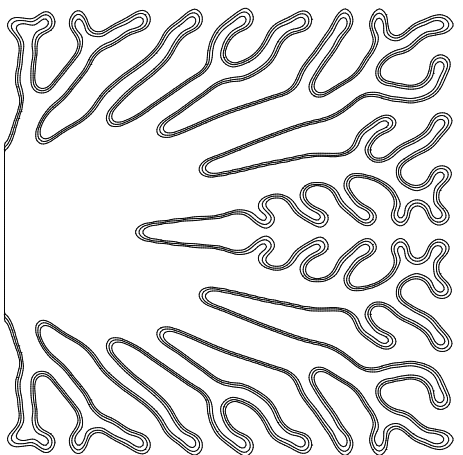

(c) $m_{f}=1.299, \sigma_{f}=0.098$.

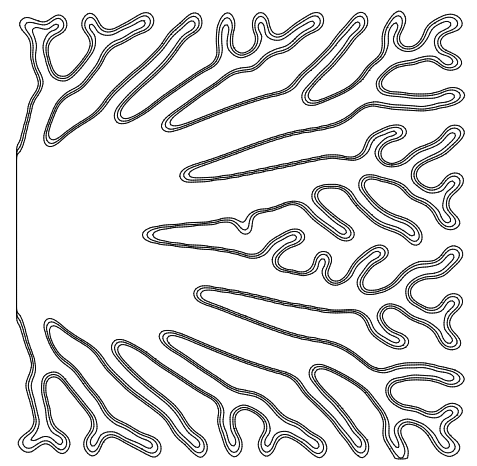

(e) $m_{f}=1.301, \sigma_{f}=0.120$.

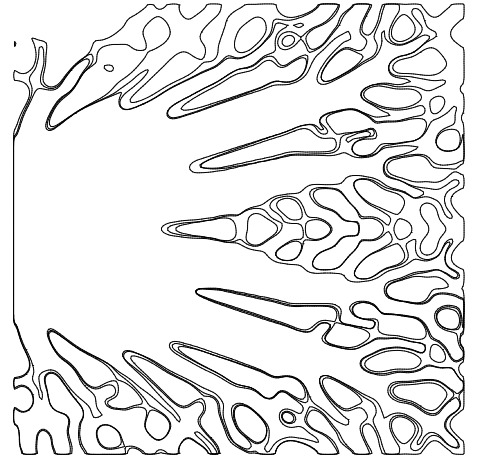

(b) $m_{f}=1.953, \sigma_{f}=0.643$.

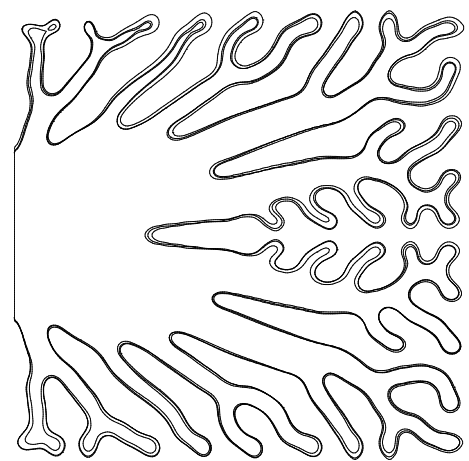

(d) $m_{f}=1.295, \sigma_{f}=0.042$

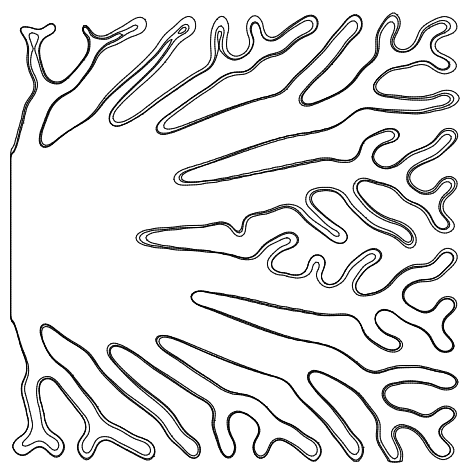

(f) $m_{f}=1.296, \sigma_{f}=0.050$.

Figure 19: The effect of uniform (left) and non-uniform (right) manufacturing errors on the optimized heat sink design obtained assuming no manufacturing errors (top), uniform manufacturing errors (middle), and non-uniform manufacturing errors (bottom). The top circle in the bottom right corner represents the target length scale $b$ (in the reference design) of both solid and void regions. The difference between the top circle and the bottom circles represents the target magnitude $\varepsilon$ of the erosion/dilation errors. 
both designs is very similar (the mean response $m_{f}$ and the standard deviation $\sigma_{f}$ are almost identical). The same holds for the effect of non-uniform manufacturing errors. It can be concluded that also for this problem, the design obtained assuming uniform errors is also robust with respect to non-uniform errors, and vice versa.

The objective function $F(\boldsymbol{\rho})=m_{f}+\kappa \sigma_{f}$ in the case of non-uniform manufacturing errors is (slightly) better for the design obtained assuming that the manufacturing errors are uniform (figure 19d) instead of non-uniform (figure 19f). This is explained by the fact that the optimization scheme does not generally yield the exact global minimum.

\section{Conclusion}

Topology optimization is a valuable tool for the design of efficient macro-, micro-, and nano-structures. However, in classical deterministic topology optimization, the effect of uncertain parameters such as manufacturing errors on the performance of the structure is not taken into account. This may lead to a design that is very sensitive to manufacturing errors. As a consequence, the performance of the actual structure may be far from optimal.

Sigmund [37] and Wang et al. [43] propose a robust approach to topology optimization where the effect of manufacturing errors is taken into account. Erosion and dilation effects are simulated by means of a projection method: a density filter is applied, followed by a Heaviside projection using a high projection threshold to simulate an erosion and a low projection threshold to simulate a dilation. Only uniform manufacturing errors are considered (i.e. constant in magnitude over the entire design domain). The optimization problem is formulated as a worst case design problem, where an eroded, an intermediate, and a dilated design are considered simultaneously.

The present paper extends this method in order to account for non-uniform manufacturing errors (i.e. with spatially varying magnitude). A probabilistic approach is followed: the Heaviside projection threshold is modeled as a random field. This random field is characterized by a marginal probability density function and a covariance function. The marginal probability density function represents the magnitude of the manufacturing error, while the covariance function determines the variation of the manufacturing error in space. The random field is modeled as a translation process [16]: it is expressed as a nonlinear transformation of a Gaussian field, which is modeled by means of an EOLE expansion [27]. The optimization problem is formulated in a probabilistic way: the objective function is defined as a weighted average of the mean value and the standard deviation of the structural performance. In each iteration of the optimization scheme, these statistics are estimated by means of a Monte Carlo simulation, considering 100 realizations of the manufacturing error. Afterwards, a more elaborate Monte Carlo simulation is performed (using 10000 realizations) to verify the results for the optimized design.

The proposed approach is applied to two test problems: the design of a compliant gripper mechanism and the design of a heat sink. In both cases, 
a robust design with respect to non-uniform manufacturing errors is obtained. Moreover, the robust topology optimization approaches discussed provide better minimum feature size control than deterministic topology optimization. Finally, it is observed that a design obtained assuming uniform manufacturing errors also performs well if the manufacturing error is actually non-uniform (for the test problems considered in this paper).

\section{Acknowledgements}

This work was financially supported by a Center of Advanced User Support (CAUS) grant from the Danish Center of Scientific Computing (DCSC), by the Elite Research Prize from the Danish Minister of Research, and by the EUROHORCs/ESF European Young Investigator Award (EURYI, http://www.esf.org/euryi) through the grant "Synthesis and topology optimization of optomechanical systems". The first author is a postdoctoral fellow of the Research Foundation-Flanders and a member of K.U.Leuven-BOF PFV/10/002 OPTEC-Optimization in Engineering Center.

\section{References}

[1] O. Amir, M.P. Bendsøe, and O. Sigmund. Approximate reanalysis in topology optimization. International Journal for Numerical Methods in Engineering, 78(12):1474-1491, 2009.

[2] O. Amir and O. Sigmund. On reducing computational effort in topology optimization: how far can we go? Structural and Multidisciplinary Optimization, 2011. Published online: http://dx.doi.org/10.1007/s00158010-0586-7.

[3] A. Ben-Tal and A. Nemirovski. Robust truss topology design via semidefinite programming. SIAM Journal on Optimization, 7(4):991-1016, 1997.

[4] M.P. Bendsøe and N. Kikuchi. Generating optimal topologies in structural design using a homogenization method. Computer Methods in Applied Mechanics and Engineering, 71:179-224, 1988.

[5] M.P. Bendsøe and O. Sigmund. Topology optimization: theory, methods and applications. Springer, Berlin, 2003.

[6] HG. Beyer and B. Sendoff. Robust optimization - a comprehensive survey. Computer Methods in Applied Mechanics and Engineering, 196:3190-3218, 2007.

[7] B. Bourdin. Filters in topology optimization. International Journal for Numerical Methods in Engineering, 50(9):2143-2158, 2001.

[8] T.E. Bruns and D.A. Tortorelli. Topology optimization of non-linear elastic structures and compliant mechanisms. Computer Methods in Applied Mechanics and Engineering, 190(26-27):3443-3459, 2001. 
[9] S.K. Chen, W. Chen, and S.H. Lee. Level set based robust shape and topology optimization under random field uncertainties. Structural and Multidisciplinary Optimization, 41(4):507-524, 2010.

[10] F. De Gournay, G. Allaire, and F. Jouve. Shape and topology optimization of the robust compliance via the level set method. ESAIM Control Optimisation and Calculus of Variations, 14(1):43-70, 2008.

[11] A. Díaz and O. Sigmund. Checkerboard patterns in layout optimization. Structural Optimization, 10(1):40-45, 1995.

[12] J.L. Doob. Stochastic processes. John Wiley \& Sons, New York, USA, 1953.

[13] J.L. Doob. The development of rigor in mathematical probability (19001950). American Mathematical Monthly, 103:586-595, 1996.

[14] T. Gerstner and M. Griebel. Numerical integration using sparse grids. Numerical Algorithms, 18(3-4):209-232, 1998.

[15] R.G. Ghanem and P.D. Spanos. Stochastic finite elements: a spectral approach. Springer-Verlag, New York, 1991.

[16] M. Grigoriu. Simulation of stationary non-Gaussian translation processes. ASCE Journal of Engineering Mechanics, 124(2):121-126, 1998.

[17] J.K. Guest, J.H. Prevost, and T. Belytschko. Achieving minimum length scale in topology optimization using nodal design variables and projection functions. International Journal for Numerical Methods in Engineering, 61(2):238-254, 2004.

[18] H. Hotelling. Analysis of a complex of statistical variables into principal components. Journal of Educational Psychology, 24(6):417-441, 1933.

[19] C.S. Jog and R.B. Haber. Stability of finite element models for distributedparameter optimization and topology design. Computer Methods in Applied Mechanics and Engineering, 130(3-4):203-226, 1996.

[20] A. Kawamoto, T. Matsumori, S. Yamasaki, T. Nomura, T. Kondoh, and S. Nishiwaki. Heaviside projection based topology optimization by a PDEfiltered scalar function. Structural and Multidisciplinary Optimization, 2010. Published online: http://dx.doi.org/10.1007/s00158-010-0602-y.

[21] G. Kharmanda, N. Olhoff, A. Mohamed, and M. Lemaire. Reliabilitybased topology optimization. Structural and Multidisciplinary Optimization, 26(5):295-307, 2004.

[22] M. Kleiber and T.D. Hien. The stochastic finite element method: basic perturbation technique and computer implementation. John Wiley \& Sons, Chichester, UK, 1992. 
[23] N. Kogiso, W. Ahn, S. Nishiwaki, K. Izui, and M. Yoshimura. Robust topology optimization for compliant mechanisms considering uncertainty of applied loads. Journal of Advanced Mechanical Design Systems and Manufacturing, 2(1):96-107, 2008.

[24] A.N. Kolmogorov. Foundations of the theory of probability. Chelsea Publishing Company, New York, 2nd English edition, 1956.

[25] B.S. Lazarov, M. Schevenels, and O. Sigmund. Robust design of largedisplacement compliant mechanisms. Submitted for publication.

[26] B.S. Lazarov, M. Schevenels, and O. Sigmund. Topology optimization taking into account geometric uncertainties using perturbation techniques. In preparation.

[27] C.C. Li and A. Der Kiureghian. Optimal discretization of random fields. ASCE Journal of Engineering Mechanics, 119(6):1136-1154, 1993.

[28] P.L. Liu and A. Der Kiureghian. Multivariate distribution models with prescribed marginals and covariances. Probabilistic Engineering Mechanics, 1(2):105-112, 1986.

[29] K. Maute and D. Frangopol. Reliability-based design of MEMS mechanisms by topology optimization. Computers \& Structures, 81(8-11):813824, 2003.

[30] R.Y. Rubinstein and D.P. Kroese. Simulation and the Monte Carlo method. John Wiley \& Sons, 2nd edition, 2008.

[31] E. Sandgren and T.M. Cameron. Robust design optimization of structures through consideration of variation. Computers \& Structures, 80(2021):1605-1613, 2002.

[32] M. Schevenels, G. Lombaert, and G. Degrande. Application of the stochastic finite element method for Gaussian and non-Gaussian systems. In ISMA2004 International Conference on Noise and Vibration Engineering, pages 3299-3314, Leuven, Belgium, September 2004.

[33] G.I. Schuëller and H.A. Jensen. Computational methods in optimization considering uncertainties - An overview. Computer Methods in Applied Mechanics and Engineering, 198:1-13, 2008.

[34] C.C. Seepersad, J.K. Allen, D.L. McDowell, and F. Mistree. Robust design of cellular materials with topological and dimensional imperfections. Journal of Mechanical Design, 128(6):1285-1297, 2006.

[35] M. Shinozuka and G. Deodatis. Simulation of stochastic processes by spectral representation. Applied Mechanics Reviews, 44(4):191-204, 1991. 
[36] O. Sigmund. Morphology-based black and white filters for topology optimization. Structural and Multidisciplinary Optimization, 33(4-5):401-424, 2007.

[37] O. Sigmund. Manufacturing tolerant topology optimization. Acta Mechanica Sinica, 25(2):227-239, 2009.

[38] O. Sigmund and J. Petersson. Numerical instabilities in topology optimization: A survey on procedures dealing with checkerboards, meshdependencies and local minima. Structural Optimization, 16(1):68-75, 1998.

[39] M. Silva, D.A. Tortorelli, J.A. Norato, C. Ha, and H.R. Bae. Component and system reliability-based topology optimization using a single-loop method. Structural and Multidisciplinary Optimization, 41(1):87-106, 2010.

[40] B. Sudret and A. Der Kiureghian. Stochastic finite element methods and reliability - A state-of-the-art report. Report UCB/SEMM-2000/08, Department of Civil \& Environmental Engineering, University of California, Berkeley, November 2000.

[41] K. Svanberg. The method of moving asymptotes - a new method for structural optimization. International Journal for Numerical Methods in Engineering, 24(2):359-373, 1987.

[42] F. Wang, J.S. Jensen, and O. Sigmund. Robust topology optimization of photonic crystal waveguides with tailored dispersion properties. Journal of the Optical Society of America B: Optical Physics, 28(3):387-397, 2011.

[43] F. Wang, B.S. Lazarov, and O. Sigmund. On projection methods, convergence and robust formulations in topology optimization. Structural and Multidisciplinary Optimization, 2011. Published online: http://dx.doi.org/10.1007/s00158-010-0602-y.

[44] R.O. Weber and P. Talkner. Some remarks on spatial correlation function models. Monthly Weather Review, 121(9):2611-2617, 1993.

[45] S.L. Xu, Y.W. Cai, and G.D. Cheng. Volume preserving nonlinear density filter based on Heaviside functions. Structural and Multidisciplinary Optimization, 41(4):495-505, 2010.

[46] A.M. Yaglom. Correlation theory of stationary and related random functions. Volume I: Basic results. Springer-Verlag, New York, USA, 1987. 